\title{
DISTRIBUTIONS IN HILBERT SPACE AND CANONICAL SYSTEMS OF OPERATORS $\left({ }^{1}\right)$
}

BY

I. E. SEGAL

\section{INTRODUCTION}

Although the algebraic features of the theory of probability distributions on an infinite-dimensional linear space generally resemble those of the familiar theory on finite-dimensional spaces, there are some important differences, in which certain of the mathematical difficulties of quantum field theory originate. In the present paper we treat one such aspect of distributions, their absolute continuity and transformation properties, and initiate the application of the results to uniqueness and classification problems arising in the theory of quantum statistics.

From a purely mathematical viewpoint, the contrast with the finite (-dimensional) situation is shown by the existence of a continuum of distinct classes of quasi-invariant distributions on an infinite-dimensional Hilbert space (i.e. distributions absolutely continuous with respect to all their translates), as opposed to the unique class in the finite case. The notion of distribution that is used here is a reformulation, which is required in order to accommodate significant concrete cases as well as for theoretical purposes, but which requires (in the infinite case) the abandonment of the idea of a distribution as a countably additive probability measure on subsets of the space. The linear and topological structure of the space roughly compensates for the loss of countable additivity, particularly in the case of Hilbert space, so that there emerges an effective theory parallel in its preliminary formal aspects to the usual one.

In quantum field theory an important part is played by self-adjoint operators $P_{1}, P_{2}, \cdots$ and $Q_{1}, Q_{2}, \ldots$ satisfying the (partially formal) relations

$$
\begin{aligned}
P_{j} P_{k}-\sigma P_{k} P_{j} & =i[(1-\sigma) / 2] \delta_{j k} I=Q_{j} Q_{k}-\sigma Q_{k} Q_{j}, \\
P_{j} Q_{k}-\sigma Q_{k} P_{j} & =i[(1+\sigma) / 2] \delta_{j k} I,
\end{aligned}
$$

where $I$ is the identity operator, $i=(-1)^{(1 / 2)}$, and $\sigma= \pm 1$, depending on whether the field obeys Bose-Einstein (symmetric) or Fermi-Dirac (skewsymmetric) statistics. The uniqueness of standard methods of "quantizing" a partial differential equation depends on the extent to which: (1) such "ca-

Presented to the Society, October 27, 1956; received by the editors October 19, 1956.

(1) Research done in part under a contract between the Office of Naval Research and the University of Chicago, and in part with support from the Guggenheim Foundation and the Institute for Advanced Study. 
nonical systems" of operators are determined by these relations (within unitary equivalence); (2) simple transformations preserving these relations ("pseudocanonical" transformations, say,- - e.g. $Q_{n} \rightarrow \alpha_{n} Q_{n}$ while $P_{m} \rightarrow \alpha_{m}^{-1} P_{m}$, for a BoseEinstein field) are implementable by unitary transformations. Answers to these questions that are fairly complete at the required level are deducible from results and methods of the theory of distributions. They show the same disparity between the finite and infinite cases,- - the uniqueness [15] and the (obvious) implementability of simple pseudo-canonical transformations by unitary transformations in the finite case contrasting with great lack of uniqueness and corresponding nonimplementability in the infinite case.

Together with the formal invariance of the standard methods of quantizing a partial differential equation under pseudo-canonical transformations such as the one described, this indicates the impossibility of effecting such quantization in a unique manner (even in the speciously simple case of free fields), except in so far as the differential equation effectively paraphrases a group representation (i.e. through the use of covariance considerations). For such reasons it is natural to attempt to reformulate quantum field theory so that it is a group representation rather than a partial differential equation that is the object to be quantized. But while this reformulation eliminates the uniqueness difficulties, it suggests the questions of the structure and existence of canonical systems in the case of interacting fields. These questions have not yet been explicitly resolved in the case of any higher-order interaction of realistic type. It therefore seems appropriate to approach them through the classification of certain types of canonical sets as undertaken below.

Absolute continuity and transformation of distributions are treated in Part I of this paper. Among earlier work that is technically related we note especially that of Kakutani [7, §3], Cameron and Martin [1] and [2, \$4], and von Neumann $[16, \S 5]$. Applications to Bose-Einstein and Fermi-Dirac fields are made in Parts II and III respectively. Another approach to similar applications has been sketched by Gårding and Wightman in [5] and [6]; cf. also Friedrichs [4]. Some of the results of Part II, in particular the nonimplementability by unitary transformations of the pseudo-canonical transformations described above, were presented in a seminar on the mathematical foundations of quantum mechanics at the Institute for Advanced Study in 1951-1952. For the physical background of our work we refer primarily to [13] and the literature cited there.

\section{Absolute continuity of distributions}

1. Preliminaries. A "distribution" on a (real) linear topological space $\mathfrak{H C}$ may be defined as an equivalence class of linear maps $m$ from a dense linear subspace $\mathfrak{K}^{*}$ of the dual $\mathfrak{F}^{*}$ of $\mathfrak{F}$, into the self-adjoint measurable operators affiliated with a ring of operators $a$ (in the sense of Murray and von Neu- 
mann), on which there is defined a distinguished probability gage $E$ (the "expectation" functional), two such maps $m$ and $n$ being equivalent when for any finite set of vectors $x_{1}, x_{2}, \cdots, x_{k}$ in $\mathfrak{K}^{*}$, the rings determined by $m\left(x_{1}\right), m\left(x_{2}\right), \cdots, m\left(x_{k}\right)$ on the one hand and by $n\left(x_{1}\right), n\left(x_{2}\right), \cdots, n\left(x_{k}\right)$ on the other, are algebraically isomorphic in a fashion that takes $m\left(x_{j}\right)$ into $n\left(x_{j}\right)(j=1,2, \cdots, k)$ and preserves expectations. It is no significant loss of generality to assume, as will always be done, that the expectation of a nonnegative operator vanishes only if the operator vanishes. The foregoing definition gives a formally slightly more general class than that treated in [12], but a distribution in the present sense gives rise to a distribution in the former sense relative to a different topology on $\mathfrak{H C}$ (namely, the weakest in which all the functionals in $\mathfrak{K}^{*}$ are continuous), so that the theory of integration with respect to distributions, as well as other aspects that are independent of the topology on the underlying space, are applicable to the present class. A distribution will frequently be denoted by a particular representative $m$ for its equivalence class. For example, the Hilbert space of all square-integrable operators with respect to such a distribution may be denoted as $L_{2}(\mathfrak{F C}, m)$.

The distributions for which the ring $a$ is abelian may equivalently be defined in terms of random variables rather than self-adjoint operators (all such random variables being defined on the same space), with $m$ equivalent to $n$ in case $m\left(x_{1}\right), m\left(x_{2}\right), \cdots, m\left(x_{k}\right)$ on the one hand and $n\left(x_{1}\right), n\left(x_{2}\right), \cdots$, $n\left(x_{k}\right)$ on the other have the same joint distribution for any finite set of vectors $x_{1}, x_{2}, \cdots, x_{k}$ in $\mathfrak{K}^{*}$. Some readers may find it advantageous to interpret the work in Part $\mathrm{I}$ in terms of this alternative definitition for the abelian case. The treatment of Fermi-Dirac fields involves the nonabelian case, but noncommutative absolute continuity considerations are significant for field theory primarily in connection with the coupling of a Bose-Einstein with a Fermi-Dirac field, which will not be treated here.

A distribution $m$ in $\mathfrak{F}$ is said to be continuous if it is everywhere defined (i.e. $\mathfrak{K}^{*}=\mathfrak{K}^{*}$ ) and if whenever $x_{\lambda} \rightarrow x$ in $\mathfrak{K}^{*}$ (relative to a specified topology, which will be taken as the strong topology when $\mathfrak{H C}$ is a Banach space), $m\left(x_{\lambda}\right) \rightarrow m(x)$ in the sense that $f\left(m\left(x_{\lambda}\right)\right) \rightarrow f(m(x))$ in $L_{2}$, for all continuous realvalued functions $f$ vanishing outside compact sets; this type of convergence of $m\left(x_{\lambda}\right)$ is called "convergence in probability" or "asymptotic convergence," as this is what it amounts to in the case of an abelian distribution defined through random variables. The distribution $m$ is said to be closed if whenever $\left\{x_{\lambda}\right\}$ is a net in the domain $\mathfrak{K}^{*}$ of $m$ that converges to an element $x$ of $\mathfrak{K}^{*}$, in such a way that $\left\{m\left(x_{\lambda}\right)\right\}$ is convergent in probability to a measurable operator $f$, then $x \in K^{*}$ and $m(x)=f$.

Example 1. Let $m$ be the canonical normal distribution on a Hilbert space $\mathcal{H}$. This is the unique (within a constant factor $c$, the variance parameter) abelian distribution on $\mathfrak{F}$ such that orthogonal vectors in $\mathcal{F C}^{*}$ (which may and will be identified with $\mathfrak{F}$, in the case of a Hilbert space) correspond 
to stochastically independent operators. Let $T$ be any closed densely-defined linear operator in $\mathfrak{F C}$. Defining $n$ as the distribution in $\mathfrak{F C}$ whose domain is that of $T$ and which is given by the equation $n(x)=m(T x)$, then $n$ is closed.

To see this, note that a sequence of normal random variables of zero mean converges in probability if and only if it converges in $L_{2}$. For suppose that $\left\{u_{n}\right\}$ is such a sequence and suppose (as is not an essential loss of generality) that $u_{n} \rightarrow 0$ in probability. Then if $\gamma>0$ and if $\sigma_{n}^{2}$ denotes the variance of $u_{n}$, $\sigma_{n}^{2} \leqq \gamma^{2}+2 \sigma_{n}^{2} \int_{\gamma}^{\infty} t^{2} \exp \left[-t^{2} /\left(2 \sigma_{n}^{2}\right)\right]\left(2 \pi \sigma_{n}^{2}\right)^{-(1 / 2)} d t$, and integration by parts leads without difficulty to the estimate $\sigma_{n}^{2} \leqq \gamma^{2}+2 \sigma_{n}^{2} \delta_{n}(\gamma)\left(\gamma^{2}+1\right)$, where $\delta_{n}(\gamma)$ is the probability that $\left|u_{n}\right|$ exceeds $\gamma$. Hence if $x_{k} \rightarrow x$ and $n\left(x_{k}\right) \rightarrow f$ in probability, with the $x_{k}$ in the domain of $T$, then automatically $n\left(x_{k}\right) \rightarrow f$ in $L_{2}$. But $|m(x)|_{2}=c|x|$, where $c$ is a constant and $|\cdot|_{2}$ indicates the norm in $L_{2}$, so this means $\left|T x_{i}-T x_{j}\right|_{2} \rightarrow 0$. Hence $x$ is in the domain of $T$, and hence of $n$, and $n(x)=f$.

It is immediate that the covariance function $C(x, y)=E[n(x) n(y)]$ of the distribution $n$ has the form $C(x, y)=(A x, y)$, where $A=T^{*} T$. It is interesting to note that on the other hand, if $A$ is any given non-negative self-adjoint operator on $\mathfrak{F}$, there exists a unique distribution $n$ in $\mathscr{H}$ with the properties: (1) the domain of $n$ is the domain of $A^{(1 / 2)}$; (2) for any finite set of vectors $x_{1}, x_{2}, \cdots, x_{k}$ in the domain of $n, n\left(x_{1}\right), n\left(x_{2}\right), \cdots, n\left(x_{k}\right)$ are jointly normally distributed with vanishing means; (3) the covariance function of $n$ is $(A x, y)$. This uniqueness follows from the fact that a (finite-dimensional) normal multivariate distribution is determined by the corresponding means and covariances, together with the definition of equivalence of distributions. The foregoing distribution may appropriately be designated as the general normal distribution in $\mathcal{H C}$ with covariance operator $A$ and vanishing mean. That with mean $a$ in $\mathfrak{H}$ and covariance operator $A$ is given by the equation $n(x)$ $=m\left(A^{(1 / 2)} x\right)-(x, a) I$, where $I$ denotes the identity operator, and $n$ has the same domain as $A^{(1 / 2)}$.

Definition 1. A distribution $m$ on a Hilbert space $\mathcal{H}$ is said to be $a b s o$ lutely continuous with respect to a distribution $n$ on $\mathfrak{H C}$ (symbolically, $m<n$ ) in case its domain contains that, $D$, of $n$ and there exists a completely additive homomorphism $\phi$ of the ring $\mathfrak{N}$ of $n$ into the ring $\mathfrak{N}$ of $m$, (with extension to the respective rings of measurable operators) taking $n(x)$ into $m(x)$, for all $x$ in $D$. The derivative of $m$ with respect to $n$, denoted $d m / d n$, is defined as the unique operator $A$ in $L_{1}(H, n)$ such that $E_{m}(\phi(X))=E_{n}(A X)$ for all operators $X$ in $\mathscr{N}$, where $E_{d}$ denotes the expectation functional for the distribution $d$.

In connection with this definition it should be remarked that in the term "homomorphism" is included the requirements that adjoints go into adjoints and the identity into the identity. "Complete additivity" of a homomorphism $\phi$ is the property $\phi\left(\mathrm{U}_{\mu} P_{\mu}\right)=\mathrm{U}_{\mu} \phi\left(P_{\mu}\right)$, where $\left\{P_{\mu}\right\}$ is any indexed family of mutually orthogonal projections (the indicated union being in the lattice of 
all projections). The most general such homomorphism is readily seen to be obtained by following the homomorphism consisting of the restricting of the operators in $N$ to the orthocomplement of the range of the maximal projection annihilated by $\phi$ by a completely additive isomorphism. It is no essential loss of generality to take the ring of any distribution to be standard (cf. $[10]$ ), and making this assumption henceforth, any such isomorphism is automatically onto (the weak closure of its image) and may be implemented by a unitary equivalence. It follows readily that $\phi$ may be extended to a homomorphism $\tilde{\phi}$ of the algebra $\mathscr{N}$ of all operators measurable with respect to $\mathscr{N}$ into the corresponding algebra $\mathbb{T}$ for $m$, that is uniquely determined by the condition that $\phi(f(A))=f(\phi(A))$ for every self-adjoint measurable operator $A$ and continuous real-valued function $f$ on the line that vanishes outside a compact set. It is therefore unambiguous and may be convenient to speak of $\phi$ as taking the self-adjoint measurable operator $A$ into $\phi(A)$, although strictly speaking it is not $\phi$ but its extension $\tilde{\phi}$ that does this.

Two distributions $m$ and $n$ are said to be equivalent in the sense of absolute continuity, or mutually absolutely continuous (symbolically, $m \sim n$ ), in case each is absolutely continuous with respect to the other. Evidently (in view of the preceding paragraph) this is the case if and only if there exists an algebraic isomorphism between $\mathscr{N}$ and $\mathscr{N}$ that takes $m(x)$ into $n(x)$ for all $x$ in a common domain. Given that $m<n$, and that the domains coincide, this is the case if and only if the central operator $d m / d n$ is invertible; the inverse is then carried by the isomorphism into $d n / d m$.

2. Approach to absolute continuity. In general in an infinite-dimensional space it may be necessary to determine absolute continuity or evaluate derivatives through reduction to the finite or other special case by means of such a result as

THEOREM 1. A distribution $m$ is absolutely continuous with respect to a distribution $n$ on a Hilbert space $\mathfrak{H C}$ if and only if

(1) its domain contains that of $n$, and if for every subspace $F$ in a cofinal directed system $\Sigma$ of subspaces of the domain of $n, m_{F}$ is absolutely continuous with respect to $n_{F}$, where $m_{F}$ and $n_{F}$ denote the respective restrictions of $m$ and $n$ to $F$;

(2) the net $\left\{d m_{F} / d n_{F} ; F \in \Sigma\right\}$ is convergent in $L_{1}(\mathfrak{H}, n)$. In the event of absolute continuity, the limit of this net is $\mathrm{dm} / \mathrm{dn}$.

We may note that the subspaces of the domain $\pi$ of $n$ are taken as directed by inclusion. It is easily seen that a directed system of such subspaces is cofinal if and only if every vector in $\nVdash$ is contained in some subspace of the system. The proof of the theorem uses an extension of the concept of conditional expectation.

Definition 2. Let $T$ be an operator that is integrable with respect to a ring $A$ and an expectation function (probability gage) $E$. The conditional ex- 
pectation of $T$ with respect to a subring $B$ of $Q$ is defined as the unique integrable operator $T^{\prime}$ affiliated with $B$ such that $E(X T)=E\left(X T^{\prime}\right)$ for all elements $X$ of $B$.

The existence of the conditional expectation, which will be denoted as $E[T \mid \Theta]$, follows from the theory of integration with respect to gages [10]. We next extend a result that is well known in the commutative case in other forms (e.g. as a martingale convergence theorem).

LEMmA 1.1. Let $\left\{a_{F} ; F \in \Sigma\right\}$ be a directed system of subrings of the ring $a$, with $a_{F} \subset Q_{G}$ for $F \subset G$, and let $Q$ be generated (as a ring) by the $a_{F}$. Then for any integrable operator $T$ affiliated with $a,\left\{E\left[T \mid a_{F}\right]: F \in \Sigma\right\}$ converges in $L_{1}(Q, E)$ to $T$.

There is no difficulty in making the estimate $|E[T \mid \beta]|_{1} \leqq|T|_{1}$, for any subring $B$, so that the operation of taking the conditional expectation is bounded by unity, with respect to the $L_{1}$ norm. It follows that it suffices to prove the convergence of the conditional expectations for a set of operators that is dense in $L_{1}(Q, E)$. Now the set-theoretic union $a_{0}$ of the $a_{F}$ is such a dense set. For otherwise, there would exist, by the Hahn-Banach theorem and the known form for the continuous linear functionals on $L_{1}(a, E)$, a nonzero element $S$ of $a$ such that $E(S X)=0$ for all $X$ in $a_{0}$. As $Q$ is standard, it may be taken to be the left ring of a Hilbert algebra (cf. [10]) and $E(S X)$ may then be represented in the form of a weakly continuous linear functional $E(S X)=\left(X I^{\prime}, S^{* \prime}\right)$, where $T^{\prime}$ denotes the element of the Hilbert algebra, left multiplication by which is the operator $T$, and $I$ denotes the identity. Now $Q_{0}$ is a self-adjoint algebra, so that as it generates $Q$ must be weakly dense in $Q$, implying that the foregoing functional must vanish identically, in contradiction with the nonzero character of $S$.

Proof of theorem. Taking the "only if" part first, (1) is obvious, so that only (2) need be shown. Putting $T=d m / d n$ and $T_{F}=d m_{F} / d n_{F}$, it is easily verified that $E\left[T \mid \mathfrak{Q}_{F}\right]=T_{F}$, where $\mathfrak{Q}_{F}$ is the ring generated by the continuous functions vanishing outside compact sets, of the $n(x)$, with $x$ varying over $F$, while with this notation $Q=a_{\mathcal{K}}$. Hence by the cofinality of $\Sigma$, the $a_{F}$ generate $Q$, and (2) follows from Lemma 1.1, as does the fact that $T=\lim _{F} T_{F}$, in $L_{1}(\mathfrak{H C}, n)$.

To prove the "if" part, let $\phi_{F}$ denote a completely additive homomorphism of the ring $\Theta_{F}$ determined by the $n(x), x$ in $F$, into the ring $Q_{F}$ determined by the $m(x), x$ in $F$, whose extension $\tilde{\phi}_{F}$ takes $n(x)$ into $m(x)$ for all $x$ in $F$. Now if $F \subset G$, the restriction of $\phi_{G}$ to $B_{F}$ is a completely additive homomorphism of $\Theta_{F}$ onto $Q_{F}$ whose extension to the ring $\mathscr{N}_{F}$ of all measurable operators with respect to $B_{F}$ is readily shown to take $n(x)$ into $m(x), x \in F$. It follows that this restriction coincides with $\phi_{F}$, so that $\phi_{F} \subset \phi_{G}$. Hence there exists an algebraic homomorphism $\phi_{0}$ of the set-theoretic union $\Theta_{0}$ of the $\Theta_{F}, F \in \Sigma$, into $Q_{0}=\bigcup_{F \in \Sigma} Q_{F}$, such that the restriction of $\phi_{0}$ to $\Theta_{F}$ is $\phi_{F}$. 
It is convenient to introduce here the following type of convergence: a sequence $\left\{X_{k}\right\}$ of elements of the ring $\Omega$ of a probability gage space is said to be "boundedly $L_{2}$-convergent" (for short, $B L_{2}$-convergent) to an element $X$ in case $\left|X_{k}-X\right| \rightarrow 0$ while $\left|X_{k}\right|_{\infty}$ (i.e. the usual operator bound) is bounded. We note that a self-adjoint subalgebra $R_{0}$ of $R$ generates $R$ if and only if it is dense in $R$ with respect to $B L_{2}$-convergence. This is proved in [8] for factors, and there is no essential difficulty in extending the result to arbitrary probability gage spaces by means of methods given in [10].

Next observe that $\phi_{0}$ is $B L_{2}$-continuous: if $\left\{X_{i}\right\} B L_{2}$-converges to zero ( $X_{i}$ in $\left.Q_{0}\right)$, then $\left\{\phi_{0}\left(X_{i}\right)\right\}$ is $B L_{2}$-convergent to zero. To see this, note that for any operator $T$ in $\Theta_{F}, E_{m}\left(\phi_{0}(T)\right)=E_{n}\left(T D_{G}\right)$, for any $G$ containing $F$ and in $\Sigma$, where $D_{G}=d m_{G} / d n_{G}$. It follows that $E_{m}\left(\phi_{0}(T)\right)=E_{n}(T D)$ for all $T$ in $B_{0}$, where $D$ is the $L_{1}$-limit of the net $\left\{D_{G} ; G \in \Sigma\right\}$. Now $\left|\phi_{0}\left(X_{i}\right)\right|_{2}^{2}$ $=E_{m}\left(\phi_{0}\left(X_{i} * X_{i}\right)\right)=E_{n}\left(X_{i} * X_{i} D\right)$. It is easy to conclude from the integrability of $D$ that $E_{n}\left(X_{i} * X_{i} D\right) \rightarrow 0$ as $i \rightarrow \infty$.

Now any operator $X$ in $B$ is the $B L_{2}$-limit of a sequence of elements of $\beta_{0}$. Hence $\phi_{0}$ may be extended to all of $\beta$ by setting $\phi(X)$ equal to the limit in $L_{2}(\mathcal{H C}, m)$ of the sequence $\left\{\phi_{0}\left(X_{i}\right)\right\}$, where $\left\{X_{i}\right\}$ is a sequence of elements of $\beta_{0}$ converging to $X$. That $\phi$ is well-defined follows from the continuity property of $\phi_{0}$ proved above. There is no difficulty in verifying that it is a homomorphism. Passing to the limit in the equation $E_{m}\left(\phi_{0}\left(X_{i}\right)\right)=E_{n}\left(X_{i} D\right)$ $\left(X_{i}\right.$ in $\left.\beta_{0}\right)$, it follows, using the definition of $\phi(X)$ and continuity properties of the expectation, that $E_{m}(\phi(X))=E_{n}(X D)$.

Now $\phi$ is $B L_{2}$-continuous, i.e. $\phi\left(X_{i}\right) \rightarrow 0$ in $L_{2}(\mathfrak{F C}, m)$ and uniformly boundedly in $L_{\infty}(\mathfrak{F C}, m)$ if $\left\{X_{i}\right\}$ is a sequence of elements of $\beta$ that is $B L_{2}$ convergent to zero. For $E_{m}\left[\phi\left(X_{i}\right) * \phi\left(X_{i}\right)\right]=E_{n}\left(X_{i} * X_{i} D\right) \rightarrow 0$. It follows in particular that $\phi$ is completely additive. To complete the proof it remains only to note that $\widetilde{\phi}(n(x))=m(x)$, by virtue of the definition of $\phi_{0}$ and the fact that $\phi$ extends $\phi_{0}$.

A stronger and substantially more applicable result than Theorem 1 is valid when the distributions involved are closed. We may recall that a collection of vectors (or submanifolds) in the domain $K$ of a closed distribution is determining in case for every vector $x$ in the domain there is a sequence $\left\{x_{k}\right\}$ of vectors in the given collection such that $x_{k} \rightarrow x$, while $n\left(x_{k}\right) \rightarrow n(x)$ in probability.

Corollary 1.1. A closed distribution $m$ is absolutely continuous with respect to a closed distribution $n$ provided conditions (1) and (2) of Theorem 1 hold with $\Sigma$ a determining (rather than cofinal) directed system of submanifolds of the domain of $n$.

The simplest type of application of this result is to the case when $\Sigma$ consists of the subspaces spanned by $e_{1}, e_{2}, \cdots, e_{k}(k=1,2, \cdots)$, where $\left\{e_{i}\right\}$ is an orthonormal basis of domain vectors for the separable Hilbert space $H$. 
This corollary is not strictly a special case of Theorem 1 , but its proof follows by the same method. Using the notation of the proof of Theorem 1, the only points where the closed character of the distributions is required are (1) the proof that the ring $B_{0}$ is strongly dense in $B ;(2)$ the proof that $\widetilde{\phi}(n(x))=m(x)$ for all $x$ in the domain $\mathcal{K}$ of $n$. For both of these it suffices to show that for every $x$ in $\mathscr{K}$ and real-valued continuous function $f$ vanishing outside a compact set, $\phi(f(x))$ is the $B L_{2}$-limit of a sequence $\left\{\phi\left(f\left(x_{i}\right)\right)\right\}$, with $x_{i}$ in a subspace $F$ in $\Sigma$. This is the case because of the determining and closed characters of $\mathscr{K}$ and $n$ respectively, together with the $B L_{2}$-continuity of $\phi_{0}$.

3. Abelian and skew distributions. A still more readily applicable result is available for the important special case of direct (resp. skew) products of abelian (resp. skew) distributions $\left({ }^{2}\right)$. This result in the abelian case is similar to that of Kakutani in [7].

THEOREM 2. Let $m$ and $n$ be closed abelian (resp. skew) distributions on a Hilbert space $\mathfrak{H}$, which is the direct sum of the subspaces $\mathfrak{H}_{\lambda}, \lambda \in \Lambda$, each of which is contained in the domain of $n$. Suppose that the restrictions of $m$ and $n$ to any finite direct sum of the $\mathfrak{H}_{\lambda}$ are the direct (resp. skew) products of the restrictions of $m$ and $n$ to the corresponding $\mathfrak{H}_{\lambda}$. Then if $m$ and $n$ are closed and the collection of all finite direct sums of the $\mathfrak{H}_{\lambda}$ is determining for them, $m$ is absolutely continuous with respect to $n$ if and only if: (1) $m_{\lambda}$ is absolutely continuous with respect to $n_{\lambda}$ for all $\lambda$ (where the subscript " $\lambda$ " indicates restriction to $H_{\lambda}$ ); (2) the product $\prod_{\lambda} E_{n_{\lambda}}\left[d m_{\lambda} / d n_{\lambda}\right]$ is convergent $\left({ }^{3}\right)$.

We require first a lemma asserting that $L_{1}$-convergence and $L_{2}$-convergence are equivalent in a certain situation.

LEMmA 2.1. Let $\left\{T_{\mu}\right\}$ be a directed system of non-negative commuting measurable operators with respect to a probability gage space, each of which has unit expectation. Then the net $\left\{T_{\mu}\right\}$ is convergent in $L_{1}$ if and only if the net $\left\{T_{\mu}^{(1 / 2)}\right\}$ is convergent in $L_{2}$.

Suppose that $\left\{T_{\mu}\right\}$ is convergent in $L_{1}$. From the inequality

$$
\left|a^{(1 / 2)}-b^{(1 / 2)}\right|^{2} \leqq|a-b|
$$

for arbitrary non-negative real numbers $a$ and $b$, it follows that $\left|S_{\mu}-S_{\nu}\right|_{2}^{2}$

${ }^{(2)}$ A distribution $m$ is defined as skew in case it is reflection-invariant (i.e. $-m(\cdot)$ represents the same distribution as $m(\cdot)$ ), and $m(x) m(y)+m(y) m(x)$ is central for all $x$ and $y$ in $\mathcal{H}$. This supersedes the definition of skew distribution given in [12], whose results are valid with the present extended definition (and not always as stated), with the exception of Theorem 3, in whose statement "skew distribution" should be replaced by "reflection-invariant distribution $m$ such that $m(x) m(y)+m(y) m(x)=0$ for orthogonal $x$ and $y$." It may be noted that abelian distributions may be correspondingly defined as those for which $m(x) m(y)-m(y) m(x)$ is central for all $x$ and $y$ in $\mathfrak{F C}$; this has been proved by R. J. Blattner.

(3) A numerical product $\prod_{x} c_{x}$ is said to be convergent here in case the net $\left\{\prod_{x \in F} c_{\lambda}\right\}$, where $F$ is an arbitrary finite set of indices, is convergent in the usual sense. 
$\leqq\left|T_{\mu}-T_{\nu}\right|_{1}$, where $S_{\mu}=T_{\mu}^{(1 / 2)}$, which implies the "if" part of the lemma. On the other hand, $\left|T_{\mu}-T_{\nu}\right|_{1}=\left|\left(S_{\mu}+S_{\nu}\right)\left(S_{\mu}-S_{\nu}\right)\right|_{1}$, as the $T_{\mu}$ commute, and applying Schwarz' inequality, it follows that $\left|T_{\mu}-T_{\nu}\right|_{1} \leqq\left|S_{\mu}+S_{\nu}\right|_{2}\left|S_{\mu}-S_{\nu}\right|_{2}$ $\leqq 4\left|S_{\mu}-S_{\nu}\right|_{2}$, from which the "only if" part follows.

Proof of theorem, Abelian case. It is clear that (1) must hold. Now let $\Sigma$ denote the collection of all finite direct sums of the $\mathcal{H}_{\lambda}, \lambda \in \Lambda$, and for any subspace $F$ in $\Sigma$ let $m_{F}$ and $n_{F}$ be as above. Then it follows that $m_{F}<n_{F}$, and in fact, putting $D_{F}=d m_{F} / d n_{F}$ and $T_{\lambda}=D \mathcal{F}_{\lambda}, D_{F}=\prod_{i=1}^{k} T_{\lambda_{i}}$, where $F$ $=\sum_{i=1}^{k} \oplus \mathcal{F}_{\lambda_{i}}$. Now by Corollary 1.1, $\left\{D_{F}\right\}$ must be convergent in $L_{1}$, which by Lemma 2.1 implies that $\left\{D_{F}^{(1 / 2)}\right\}$ is convergent in $L_{2}$.

Now consider $\left|D_{F}^{(1 / 2)}-D_{G}^{(1 / 2)}\right|_{2}$, where $G=\sum_{i=1}^{j} \oplus \mathcal{F C}_{\lambda_{i}}$, with $j<k$. It is readily computed that $\left|D_{F}^{(1 / 2)}-D_{G}^{(1 / 2)}\right|=2-2 \operatorname{Re}\left\{\prod_{i=j+1}^{k} E\left[T_{\lambda_{i}}^{(1 / 2)}\right]\right\}$. Hence $\operatorname{Re}\left\{\prod_{i=j+1}^{k} E_{n}\left[T_{\lambda_{i}}^{(1 / 2)}\right]\right\} \rightarrow 1$ as $j, k \rightarrow \infty$, which means, noting that $\left|E_{n}\left(T_{\lambda}^{(1 / 2)}\right)\right|$ $\leqq 1$, that the product $\prod_{\lambda} E_{n}\left[T_{\lambda}^{(1 / 2)}\right]$ is convergent.

Now assume conversely that (1) and (2) hold. Reversing the argument just made and using the converse part of Lemma 2 shows that $\left\{D_{F}\right\}$ is a convergent net in $L_{1}$. It follows from Corollary 1.1 that $m<n$.

Proof of theorem, skew case. In connection with this part of the proof we make

Definition 3. Let $m_{i}(i=1,2)$ be skew distributions on the Hilbert spaces $\mathfrak{H}_{i}$ with skew product $m$ on $\mathfrak{F}=\mathcal{F}_{1} \oplus \mathcal{F}_{2}$. Let $T_{i}$ be measurable with respect to the ring of $m_{i}$. The skew product $T_{1} \tilde{X} T_{2}$ of $T_{1}$ with $T_{2}$ is defined as the operator $T$ measurable with respect to the ring of $m$ that is given by the equation $T=T_{1}^{\prime} T_{2}^{\prime}$, where $T_{i}^{\prime}$ is the image of $T_{i}$ under the (unique) completely additive isomorphism of the measurable operators with respect to $m_{i}$ into the measurable operators with respect to $m$ that takes $m_{i}(x)$ into $m(x)$.

LEMma 2.2. The skew product of operators has the form

$$
2 T_{1} \tilde{\times} T_{2}=T_{1} \times\left(T_{2}+\Omega_{2} T_{2} \Omega_{2}\right)+\Omega_{1} \times\left(T_{2}-\Omega_{2} T_{2} \Omega_{2}\right)
$$

relative to the representative for the skew product of distributions given by the equation $m\left(x_{1}, x_{2}\right)=m_{1}\left(x_{1}\right) \times I_{2}+\Omega_{1} \times m_{2}\left(x_{2}\right)$, where $\Omega_{i}$ is the unitary operator on $L_{2}\left(\mathfrak{F C}_{i}, m_{i}\right)$ induced by reflection $x \rightarrow-x$ on $\mathfrak{F C}_{i}$.

Using the notation of the definition of skew product, $T_{1}^{\prime}=T_{1} \times I_{2}$, for the map $T_{1} \rightarrow T_{1} \times I_{2}$ is a countably additive isomorphism of the ring of measurable operators with respect to $m_{1}$ into the corresponding ring for $m$, taking $m_{1}(x)$ into $m(x)$, for all $x$ in $\mathfrak{F C}_{1}$. On the other hand, for corresponding reasons, $2 T_{2}^{\prime}=I_{1} \times\left(\Omega_{2} T_{2} \Omega_{2}+T_{2}\right)+\Omega_{1} \times\left(T_{2}-\Omega_{2} T_{2} \Omega_{2}\right)$, and the lemma follows directly.

Lemma 2.3. Let $m_{i}$ and $n_{i}$ be skew distributions with $m_{i}<n_{i}(i=1,2)$. Then the skew product $m$ of $m_{1}$ with $m_{2}$ is absolutely continuous with respect to the skew product of $n_{1}$ and $n_{2}$, and $d m / d n$ is the skew product of $d m_{1} / d n_{i}$ and $d m_{2} / d n_{2}$.

Using the factorization of an arbitrary completely additive homomorphism of the ring of a probability gage space onto another such ring that is 
given above, as the product of a restricting to an invariant subspace with an isomorphism, it is readily seen that it suffices to prove the lemma for the case when $m_{i}$ and $n_{i}$ are equivalent in the sense of absolute continuity $(i=1,2)$. Then there exists a unitary transformation $W_{i}$ from the Hilbert space on which $R_{n_{i}}$ acts to that on which $R_{m_{i}}$ acts, where $\Omega_{d}$ denotes the ring of the distribution $d$, that implements the isomorphism corresponding to the absolute continuity $(i=1,2)$. Now define $W$ by the equation $2 W$ $=W_{1} \times\left(\Omega_{2} W_{2} \Omega_{2}+W_{2}\right)+I_{1} \times\left(W_{2}-\Omega_{2} W_{2} \Omega_{2}\right)$. There is no difficulty in verifying that $W$ is unitary and induces the transformation of the skew product $T_{1} \tilde{X} T_{2}$ of elements of $R_{n_{1}}$ and $R_{n_{2}}$ into the skew products of $\phi_{1}\left(T_{1}\right)$ and $\phi_{2}\left(T_{2}\right)$, where $\phi_{i}$ is the isomorphism of $R_{n_{i}}$ onto $R_{m_{i}}$ given by the hypothesis. It follows that there exists a completely additive homomorphism of $R_{n}$ onto $R_{m}$ that takes $n(x)$ into $m(x), x \in \mathfrak{F}_{1} \oplus \mathcal{H}_{2}$.

To evaluate $d m / d n,=D$ say, note that for arbitrary $X_{i}$ in $R_{n_{i}}$, $E\left[\phi\left(X_{1} \tilde{X} X_{2}\right)\right]=E\left[\phi_{1}\left(X_{1}\right) \tilde{X} \phi_{2}\left(X_{2}\right)\right]=E\left[\phi_{1}\left(X_{1}\right)\right] E\left[\phi_{2}\left(X_{2}\right)\right]=E\left(X_{1} D_{1}\right) E\left(X_{2} D_{2}\right)$ $=E\left(X_{1} D_{1} \tilde{X} X_{2} D_{2}\right)$, where the gages relative to which the respective expectations are formed are indicated by the context. Now using the commutativity of $D_{i}$ with $\Omega_{i}$, which results from the reflection-invariance of $m_{i}$ and $n_{i}$, it follows readily from Lemma 2.2 that $X_{1} D_{1} \tilde{\mathrm{X}} X_{2} D_{2}=\left(X_{1} \tilde{\mathrm{X}} X_{2}\right)\left(D_{1} \tilde{\mathrm{X}} D_{2}\right)$. To conclude the proof of the lemma it is only necessary to show that the skew products $X_{1} \tilde{X} X_{2}$ generate the ring of $n_{1} \tilde{X} n_{2}$. Now this ring is generated by the real-valued continuous functions vanishing outside of compact sets, of the $n_{1}\left(x_{1}\right) \times I_{2}+\Omega_{1} \times n_{2}\left(x_{2}\right), x_{i} \in H_{i}$. But if $A$ and $B$ are measurable operaters and if $f$ is continuous and vanishes outside a compact set, $f(A+B)$ is in the ring generated by $A$ and $B$, for it commutes with all unitary operators commuting with both $A$ and $B$. Hence we may restrict ourselves to the products of continuous functions vanishing outside of compact sets, of the $n_{1}\left(x_{1}\right) \times I_{2}$ and of the $\Omega_{1} \times n_{2}\left(x_{2}\right)$, showing that the products $X_{1} \tilde{X} X_{2}$ generate.

The proof for the skew case may now be completed along the same lines as the proof in the abelian case, once it is noted that the derivatives $d m_{F} / d n_{F}$ are mutually commutative, for they are skew products of the reflectioninvariant operators $d m_{\lambda} / d n_{\lambda}$, and the skew product of reflection-invariant operators is identical with their direct product, in the particular representation of the skew product employed above.

4. Affine transformations relative to the normal distribution. As an illustration of the foregoing and for its intrinsic interest we treat in this section the absolute continuity properties of the transform of the canonical normal distribution under the affine transformation $x \rightarrow T x+a$, where $T$ is a given operator and $a$ is a given vector. Our results may be compared with those of Cameron and Martin [1] and [2], which are related to ours via the representation of Brownian motion in terms of the canonical normal distribution that is noted in [11]. For simplicity of expression of the derivative we use in this section the random variable formulation of distributions.

The manner in which results such as those of Cameron and Martin may 
be derived from the present work may be illustrated by examining the case of pure translations. Let $C$ denote the space of all continuous real-valued functions on the interval $[0,1]$ that vanish at 0 , relative to Wiener measure. Then if $k$ is an absolutely continuous element of $C$, with $k^{\prime}$ in $L_{2}(0,1)$, the transformation $x(t) \rightarrow x(t)+k(t)$ on $C$ is absolutely continuous with respect to Wiener measure. This extends [1], in which it is assumed that $k^{\prime}$ is of bounded variation, and the form of the derivative shows that the present result is best possible. To prove it observe: (1) There exists a unique unitary multiplicative transformation $U$ from $L_{2}(H, n)$ onto $L_{2}(C)$ that takes $n(f)$ in to $\int_{0}^{1} f(t) d x(t)$, where $H=L_{2}(0,1)$, and $n$ is the canonical normal distribution on $H$. (2) Any such transformation between the spaces of square-integrable random variables over probability spaces is uniquely extendable to an isomorphism between the corresponding rings of all random variables that preserves expectation. (3) The equivalence of the canonical normal distribution to any of its translates, in the sense of absolute continuity, means that for any $h$ in $H$, there exists a unique isomorphism $V_{h}$ of the ring of all random variables over $H$ with respect to $n$ that takes $n(f)$ into $n(f)+(f, h)$, where $(f, h)$ $=\int_{0}^{1} f(t) h(t) d t$. (4) $U V_{h} U^{-1}$ is then an isomorphism of the ring of all random variables over $C$ that is uniquely determined by the property that it carries $\int_{0}^{1} f(t) d x(t)$ into $\int_{0}^{1} f(t) d x(t)+(f, h)$, for all $f$ in $H$. (5) If $h=k^{\prime}, \int_{0}^{1} f(t) d x(t)$ $+(f, h)=\int_{0}^{1} f(t) d[x(t)+k(t)]$. The derivative given in [1] transforms under $U$ into the derivative given by Theorem 3 for this special case.

Linear transformations may be similarly treated. For clarity of the idea we limit ourselves to transformations of the type

$$
x(t) \rightarrow x(t)+\int_{0}^{1} K(t, s) x(s) d s
$$

with $K(t, s)$ continuous; the more general case in which $K(t, s)$ has a jump at $t=s$ can be similarly treated. If $K(t, s)$ is absolutely continuous as a function of $t$ for almost all fixed $s$, and if $\int_{0}^{1} \int_{0}^{1}\left|K_{t}(t, s)\right|{ }^{2} d s d t$ is finite, then the foregoing transformation of $C$ into $C$ is absolutely continuous. For $K_{t}(t, s)$ is then a Hilbert-Schmidt kernel, so that Theorem 3 applies to the transformation: $f(t) \rightarrow f(t)+\int_{0}^{1} K_{s}(s, t) f(s) d s$, while on the other hand

$$
\int_{0}^{1}\left[f(t)+\int_{0}^{1} K_{s}(s, t) f(s) d s\right] d x(t)=\int_{0}^{1} f(t) d_{t}\left[x(t)+\int_{0}^{1} K(t, s) d x(s)\right] .
$$

This extends the corresponding result of [2], in which considerably more is assumed about $K$, and again the form of the derivative shows that the result is in a sense best possible.

TheOREM 3. For any closed densely-defined linear transformation $T$ on the Hilbert space $\mathcal{H C}$ with dense range, the transform by $T$ of the canonical normal distribution $n$ on $\mathfrak{H C}$ is absolutely continuous with respect to $n$ if and only if 
$\left(T^{*} T\right)^{(1 / 2)}$ is nonsingular and has the form $I+B$, where $B^{2}$ is an operator with absolutely convergent trace. In the latter event the normal distribution with covariance operator $A=T^{*} T$ and general mean $a$ in $H$ is likewise absolutely continuous with respect to $n$, with derivative expressible in case $B$ has absolutely convergent trace as

$$
(\operatorname{det} A)^{-(1 / 2)} \exp \left[-\left\{\left(\left(A^{-1}-I\right) x, x\right)-2\left(A^{-1} x, a\right)+\left(A^{-1} a, a\right)\right\} / 2\right] .
$$

The derivative, say $\mu(x)$, is given here as a function on $\mathfrak{F}$; strictly speaking, it should be formulated as a measurable function with respect to the distribution $n$. The simplicity of the function is however such that its proper interpretation as a random variable is clear, and it may for example be defined as the limit in mean of the net of tame functions $\left\{\mu\left(P_{F} x\right)\right\}$ as $F \rightarrow \mathfrak{H C}$, where $F$ is an arbitrary finite-dimensional linear manifold left invariant by $B$, and $P_{F}$ denotes the operation of projection onto $F$. The determinant, $\operatorname{det} A$, may of course be defined as the product of all the proper values of $A$ (each taken with its multiplicity), the convergence of this product following directly from the hypothesis.

To prove the "if" part, take a basis $\left\{e_{i}\right\}$ of proper vectors for $T^{*} T$, and apply Theorem 2 , which asserts that $m<n$, where $m$ denotes the normal distribution with covariance operator $A$ and mean 0 , and the canonical normal distribution $n$ is taken as having covariance operator $c I$, provided the following product is convergent:

$$
\begin{aligned}
\prod_{i=1}^{\infty} \int_{-\infty}^{\infty}\left\{\left[\frac{1}{\left(2 \pi \lambda_{i}\right)^{1 / 2}} \exp (\right.\right. & \left.\left.-\frac{1}{2 \lambda_{i}}\left(x-a_{i}\right)^{2}\right)\right] \\
& \left.\cdot\left[\frac{1}{(2 \pi c)^{1 / 2}} \exp \left(-\frac{x^{2}}{2 c}\right)\right]^{-1}\right\}^{1 / 2} \exp \left(-\frac{x^{2}}{2 c}\right) \frac{d x}{(2 \pi c)^{1 / 2}}
\end{aligned}
$$

here $A e_{i}=\lambda_{i} e_{i}$ and $a_{i}=\left(a, e_{i}\right)$. The $i$ th factor in this product is readily evaluated as $\left[4 \lambda_{i}^{\prime} /\left(1+\lambda_{i}^{\prime}\right)^{2}\right]^{1 / 4} \exp \left[-a_{i}^{2} / 4\left(1+\lambda_{i}^{\prime}\right)\right], \lambda_{i}^{\prime}=\lambda_{i} / c$, and putting $\lambda_{i}^{(1 / 2)}$ $=1+\delta_{i}$ and estimating for small $\delta_{i}$, it is straightforward to verify that the convergence of $\sum_{i} \delta_{i}^{2}$ and of $\sum_{i} a_{i}^{2}$ imply that of the product. For future reference we note that conversely the convergence of the product implies that of $\sum_{i} \delta_{i}^{2}$. The expression for the derivative is precisely that given by Theorem 2 , using the well-known form for the normal distribution in a finite number of dimensions.

Now we turn to the "only if" part. We show first that it suffices to consider the case when $A$ is bounded. For otherwise the restrictions of $m$ to the spectral manifolds of $A$ on which $A$ is bounded must be absolutely continuous with respect to the corresponding restrictions of $n$. Hence if the result were established in the bounded case it would follow that the restriction of $A$ to any spectral manifold on which it is bounded has the form $I^{\prime}+B^{\prime}$, with $I^{\prime}$ the identity relative to the submanifold and $B^{\prime}$ an operator with absolutely 
convergent trace. This implies that either $A$ itself has the stated form, or else $A$ has a sequence of proper values tending to infinity, which violates the condition $\sum_{i} \delta_{i}^{2}<\infty$ obtained above (restricting to the submanifold spanned by the proper vectors corresponding to the proper values forming the sequence tending to infinity).

So now suppose that $A$ is bounded. We observe next that $A$ has vacuous continuous spectrum. For otherwise, restricting to the spectral manifold determined by the continuous spectrum, we could assume that $A$ has pure continuous spectrum. The spectral theorem then shows that the group of all orthogonal transformations on $H$ that commute with $A$ leaves invariant no submanifold of finite positive dimension. Since the derivative of $m$ with respect to $n$ must clearly be invariant under this group, it must be constant by Theorem 1 of [14], contradicting the assumption of pure continuous spectrum. Thus $A$ has pure discrete spectrum. Choosing a basis of proper vectors, forming the same infinite product as above, and recalling the necessity of the condition $\sum_{i} \delta_{i}^{2}<\infty$ for convergence, it follows that $A$ has the stated form.

Now defining a "quasi-invariant" distribution on a linear space as an abelian one that is equivalent in the sense of absolute continuity to all of its translates, we may state

Corollary 3.1. On an infinite-dimensional Hilbert space there exist at least continuum many inequivalent quasi-invariant distributions.

In fact, the distributions making up this continuum may be taken to be extremely smooth, as Theorem 3 implies directly that the canonical normal distributions with varying variance parameters are all quasi-invariant and mutually inequivalent.

5. Distributions and infinite direct products of Hilbert spaces. To deal with a certain class of transformation questions for the distributions considered in $\S 3$, it is convenient to relate them to von Neumann's concept [16] of infinite direct product of Hilbert spaces with distinguished unit vectors (which in field theory play the role of vacuum states). For present purposes we may reformulate the notion as

Definition 4 . For each index $\lambda$ in the set $\Lambda$, let $z_{\lambda}$ be a unit vector in the complex Hilbert space $K_{\lambda}$. A system $\left(\mathscr{K}, z, \zeta_{F}\right)$ consisting of a complex Hilbert space $\mathscr{K}$, a unit vector $z$ in $\mathscr{K}$, and for each finite set $F$ of indices, an isometric map $\zeta_{F}$ of the direct product $\otimes_{\lambda \in F} \mathcal{K}_{\lambda}$ into $\mathcal{K}$, is called an (infinite) direct product of the $\left(\mathfrak{K}_{\lambda}, z_{\lambda}\right)(\lambda \in \Lambda)$ in case: (1) if $F \subset G$ and if $x_{\lambda}=z_{\lambda}$ for $\lambda \in G-F$, then $\zeta_{G} \otimes_{\lambda \in G} x_{\lambda}=\zeta_{F} \otimes_{\lambda \in F} x_{\lambda}$ (the indicated multiplication of vectors being with respect to the direct product); (2) $\zeta_{F} \otimes_{\lambda \in F} z_{\lambda}=z$, for all $F$; (3) the ranges of the $\zeta_{F}$ span $K$.

A direct product always exists and is unique within unitary equivalence (in the sense that for any two such systems, there is a unitary transformation between the corresponding $\mathcal{K}$ 's that exchanges the $z$ 's and the $\zeta_{F}$ 's. The proof 
of this may be inferred from [16] or given directly through the use of direct limit techniques (cf. e.g. the proof of Theorem 1 in [12]). The relevance of this variety of product to distributions is due to the fact that if $m$ is a closed abelian (resp. skew) distribution on a Hilbert space $\mathfrak{H C}$, and if the $\mathfrak{F C}_{\lambda}$ are as in Theorem 2, then the map $\zeta_{F}^{\prime}: u_{\lambda_{1}} \otimes \cdots \otimes u_{\lambda_{n}} \rightarrow u_{\lambda_{1}} \cdots u_{\lambda_{n}}$ (where the $u_{\lambda}$ are arbitrary in $L_{2}\left(\mathcal{F C}_{\lambda}, m_{\lambda}\right)$ and $F$ is the set $\left.\lambda_{1}, \cdots, \lambda_{n}\right)$ has a unique isometric extension to $\otimes_{t=1}^{n} L_{2}\left(\mathcal{F C}_{\lambda_{i}}, m_{\lambda_{i}}\right)$ and $\left(L_{2}(\mathcal{H C}, m), I, \zeta_{F}\right)$ is the direct product of the $\left(L_{2}\left(\mathfrak{F C}_{\lambda}, m_{\lambda}\right), I\right)$. The proof involves no essential novelty and may be omitted.

The following theorem, which can be compared with results in [16] may be regarded as giving a necessary and sufficient condition that an infinite product of automorphisms of respective rings of all bounded operators on an infinite set of Hilbert spaces, be convergent.

TheOREM 4. Let $\left(\mathcal{K}, z, \zeta_{F}\right)$ be the direct product of the $\left(\mathcal{K}_{\lambda}, z_{\lambda}\right)(\lambda \in \Lambda)$, and let $A_{\lambda}$ be an automorphism of the ring of all bounded linear operators on $\mathfrak{K}_{\lambda}$. Then there exists an automorphism $A$ of all bounded operators on $\nVdash$ that agrees with $\zeta_{\lambda} A_{\lambda} \zeta_{\lambda}^{-1}$ on $\zeta_{\lambda}\left(\digamma_{\lambda}\right)$ if and only if the (numerical) product $\prod_{\lambda}\left|\left(A_{\lambda}\left(P_{\lambda}\right) z_{\lambda}, z_{\lambda}\right)\right|$ is convergent, where $P_{\lambda}$ denotes the projection of $\mathfrak{K}_{\lambda}$ onto the one-dimensional subspace spanned by $z_{\lambda}$.

Note first that if $x_{\lambda} \in \Re_{\lambda},\left|x_{\lambda}\right|=1$, a necessary and sufficient condition that $\zeta_{F} \otimes_{\lambda \in F} x_{\lambda}$ converge with $F$ (to an element of $\mathscr{K}$ ) is that the product $\Pi_{\lambda}\left(x_{\lambda}, z_{\lambda}\right)$ be convergent, For if $F \subset G$,

$$
\left|\zeta_{F} \otimes_{\lambda \in F} x_{\lambda}-\zeta_{G} \otimes_{\lambda \in G} x_{\lambda}\right|=2-2 \operatorname{Re}\left\{\prod_{\lambda \in G-F}\left(x_{\lambda}, z_{\lambda}\right)\right\}
$$

must tend to unity as $F$ and $G$ increase arbitrarily. But as $\left|\prod_{\lambda \in G-F}\left(x_{\lambda}, z_{\lambda}\right)\right|$ $\leqq 1$, this implies that $\operatorname{Im}\left\{\prod_{\lambda \in G-F}\left(x_{\lambda}, z_{\lambda}\right)\right\} \rightarrow 0$, so that $\prod_{\lambda \in G-F}\left(x_{\lambda}, z_{\lambda}\right) \rightarrow 1$, i.e. the product is convergent. In the event of convergence the limit may be designated as $\prod_{\lambda} x_{\lambda}$, which indicated product may then also be called convergent.

It is easily verified that if $T_{\lambda}$ is a continuous linear operator on $\mathfrak{K}_{\lambda}$ with $T_{\lambda}=I_{\lambda}$ for all but a finite number of $\lambda$, then there exists a unique continuous linear operator $T$ on $K$ such that $T \zeta_{F}\left(u_{\lambda_{1}} \otimes \cdots \otimes u_{\lambda_{j}}\right)=\zeta_{F}\left(T_{\lambda_{1}} u_{1} \otimes \cdots\right.$ $\left.\otimes T_{\lambda_{j}} u_{\lambda_{j}}\right)$. This operator may be designated $\prod_{\lambda} T_{\lambda}$. Next observe that if $V_{\lambda}$ is a given unitary operator on $\mathfrak{K}_{\lambda}$, there exists a unitary operator $V$ on $K$ such that $V \prod_{\lambda} x_{\lambda}=\prod_{\lambda} V_{\lambda} x_{\lambda}$ for all $\prod_{\lambda} x_{\lambda}$ in which $x_{\lambda}=z_{\lambda}$ except for a finite set of $\lambda$, if and only if the numerical product $\Pi_{\lambda}\left(V_{\lambda} z_{\lambda}, z_{\lambda}\right)$ is convergent. The necessity is clear from the preceding paragraph together with the fact that $\prod_{\lambda} z_{\lambda}$ converges. To show the sufficiency, observe that $\prod_{\lambda} V_{\lambda} x_{\lambda}$ is convergent in case $\prod_{\lambda}\left(V_{\lambda} x_{\lambda}, z_{\lambda}\right)$ is convergent, and this product is identical with the product $\prod_{\lambda}\left(V_{\lambda} z_{\lambda}, z_{\lambda}\right)$ except for a finite number of factors. When such an operator $V$ exists, it may be denoted as $\Pi_{\lambda} V_{\lambda}$, which product may be called 
convergent. It should be noted that $\prod_{\lambda} V_{\lambda}$ is the strong limit of the net $\prod_{\lambda \in F} V_{\lambda}$, as the finite subset $F$ of the index set increases arbitrarily, where II $\prod_{\lambda \in F} V_{\lambda}$ denotes $\prod_{\lambda} V_{\lambda}^{\prime}$ with $V_{\lambda}^{\prime}=I_{\lambda}$ for $\lambda$ not in $F$. We note also the following readily proved formulas: if $\prod_{\lambda} S_{\lambda}$ and $\prod_{\lambda} T_{\lambda}$ are convergent products of unitary operators, then $\Pi_{\lambda}\left(S_{\lambda} T_{\lambda}\right)$ and $\prod_{\lambda} S_{\lambda}^{*}$ are convergent and $\left(\prod_{\lambda} S_{\lambda}\right)\left(\prod_{\lambda} T_{\lambda}\right)=\prod_{\lambda}\left(S_{\lambda} T_{\lambda}\right)$, while $\left(\coprod_{\lambda} S_{\lambda}\right)^{*}=\prod_{\lambda} S_{\lambda}^{*}$.

Now any automorphism of all bounded operators on a Hilbert space is induced by some unitary transformation on the space which is unique within multiplication by a scalar. Hence if an automorphism $A$ with the stated property exists, it is induced by some unitary operator $U$ such that $U *\left(\prod_{\lambda} V_{\lambda}\right) U=\prod_{\lambda} A_{\lambda}\left(V_{\lambda}\right)$ for any convergent product $\prod_{\lambda} V_{\lambda}$ of unitary operators. Putting $V_{\lambda}=i-(i-1) P_{\lambda}$, which is a unitary operator, then $V_{\lambda} z_{\lambda}$ $=z_{\lambda}$, so that $\prod_{\lambda} V_{\lambda}$ is convergent, implying that $\prod_{\lambda} A_{\lambda}\left(V_{\lambda}\right)$ is also convergent, which in turn implies the convergence of the numerical product $\prod_{\lambda}\left(A_{\lambda}\left(V_{\lambda}\right) z_{\lambda}, z_{\lambda}\right)$. The $\lambda$ th factor in the latter product is $1+(i-1)$ $\cdot\left(1-\left(A_{\lambda}\left(P_{\lambda}\right) z_{\lambda}, z_{\lambda}\right)\right)$, and using the fact that the product $\prod_{\lambda}\left(1+(i-1) \sigma_{\lambda}\right)$ is convergent with $0 \leqq \sigma_{\lambda} \leqq 1$ if and only if $\sum_{\lambda} \sigma_{\lambda}$ is convergent, it follows that $\sum_{\lambda}\left(1-\left(A_{\lambda}\left(P_{\lambda}\right) z_{\lambda}, z_{\lambda}\right)\right)$ is convergent. This implies that the given product is likewise convergent.

To see the converse, let $U_{\lambda}$ be a unitary operator on $\mathfrak{K}_{\lambda}$ that induces the given automorphism $A_{\lambda}$ and is such that $\left(U_{\lambda} z_{\lambda}, z_{\lambda}\right) \geqq 0$. Then the given product is termwise the square of the product $\prod_{\lambda}\left(U_{\lambda}, z_{\lambda}, z_{\lambda}\right)$. It follows that $\prod_{\lambda} U_{\lambda}$ exists and it is clear that this unitary operator induces an automorphism $A$ with the stated property.

\section{Bose-Einstein CANonical systems}

1. Quasi-invariant distributions and canonical systems. A "formal BoseEinstein canonical system" may be defined as pair of indexed families $P_{1}, P_{2}, \cdots$ and $Q_{1}, Q_{2}, \cdots$ of operators on a linear vector space such that the commutation relations

$$
P_{j} P_{k}-P_{k} P_{j}=0=Q_{j} Q_{k}-Q_{k} Q_{j}, \quad P_{j} Q_{k}-Q_{k} P_{j}=i \delta_{j k} I
$$

are satisfied. From the viewpoint of field theory, $\left(2^{-1}\right)^{1 / 2}\left(P_{j}+i Q_{j}\right)$ is the "creation" operator $\left.{ }^{4}\right)$ for a particle with a certain wave function $e_{j}$. The $e_{j}$ span a Hilbert space $\mathfrak{K C}$, of all "one-particle" states, and a canonical set as just defined thus depends on the choice of a basis in $\mathfrak{H}$. To arrive at a definition that is formally equivalent and independent of a basis, one needs to postulate a creation operator for a particle with arbitrary wave function $x$ in 3 . This leads to what may be described as an invariant formal Bose-Einstein canonical set, which is defined as a pair of linear maps $x \rightarrow P(x)$ and $x \rightarrow Q(x)$ from a real Hilbert space $\mathfrak{H C}$ to the elements of an algebra as before, with the commutation relations

(4) See, e.g., $\left[11\right.$, p. 108], where however $n^{(1 / 2)}$ should be replaced by $(n+1)^{(1 / 2)}$ throughout. 


$$
\begin{aligned}
P(x) P(y)-P(y) P(x) & =0=Q(x) Q(y)-Q(y) Q(x), \\
P(x) Q(y)-Q(y) P(x) & =i(x, y) I .
\end{aligned}
$$

(The reality of $\mathfrak{H C}$ is not significant; it arises from the physical requirement of invariance under charge conjugation, which need not be examined here.) However, for well-known reasons arising from difficulties with unbounded operators (cf. e.g. von Neumann [15]), these relations are still technically inappropriate, but become so when transformed by a device due to Weyl into corresponding relationships for the unitary operators $U(x)=\exp (i Q(x))$ and $V^{\prime}(x)=\exp (i P(x))$. These relations are: $U(x+y)=U(x) U(y) ; V(x+y)$ $=V(x) V(y) ; U(-x) V(-y) U(x) V(y)=e^{i(x, y)} I$ ( $x$ and $y$ arbitrary in $\mathfrak{F}$ ).

Both for theoretical purposes and for application to interacting quantum fields it appears appropriate to require something less than ordinary continuity of the $U(\cdot)$ and $V(\cdot)$, although a certain degree of continuity is essential. The relevant topology is that whose open sets are those that meet every finite-dimensional subspace in a set open in the relative (euclidean) topology. The way this arises in field theory is that the $U$ and $V$ for interacting fields (assuming their existence) arise from those $U_{0}$ and $V_{0}$ for the corresponding free (uncoupled) fields as follows: $U(x)=\lim _{n} S_{n}^{-1} U_{0}(x) S_{n}, V(x)$ $=\lim _{n} S_{n}^{-1} V(x) S_{n}$, where $\left\{S_{n}\right\}$ is a sequence of unitary operators. The $U_{0}$ and $V_{0}$, which are given relatively explicitly, are continuous on $\mathcal{H}$ in the norm topology on $\mathfrak{F}$, but all that can be asserted about $U$ and $V$, which in general can not be explicitly evaluated, is that their restrictions to arbitrary finitedimensional subspaces are continuous. Hence we make

Definition 5. A Bose-Einstein canonical system over a Hilbert space $\mathfrak{H}$ is a pair $(U, V)$ of unitary representations of the additive group of $\mathfrak{H C}$ on a complex Hilbert space $\mathfrak{K}$, that have continuous restrictions to every finitedimensional subspace and satisfy the relations $U(x) V(y) U(-x) V(-y)$ $=e^{i(x, y)} I$. The mappings $P$ and $Q$ from $\mathcal{H}$ to the self-adjoint operators on $\mathcal{K}$ that are defined by the equations $U(t x)=\exp (i Q(x)), V(t x)=\exp (i P(x))$, $-\infty<t<\infty$, are called an infinitesimal Bose-Einstein canonical system over $\mathfrak{T C}$.

The main purpose of this section is to relate such canonical systems to quasi-invariant distributions. To formulate this relation we need

Definition 6. Let $T(\cdot)$ be a unitary representation of a group $G$ on a complex Hilbert space $\mathcal{K}$. A multiplier for $T$ is a function $W(\cdot)$ on $G$ to the unitary operators on $\mathcal{K}$ such that $T(\cdot) W(\cdot)$ is again a representation of $G$. The multiplier is said to be relative to a ring $Q$ of operators on $\mathscr{K}$ in case the values of $W(\cdot)$ commute with all the elements of $a$, and two multipliers are equivalent relative to $Q$ if they are unitarily equivalent via a unitary operator that commutes with all the elements of $a$.

The following result reduces the classification of Bose-Einstein canonical systems to that of the classification of multipliers, together with the classification of quasi-invariant distributions, within the type of equivalence just defined, and absolute continuity, respectively. We treat here only the case 
when the representation space $\mathfrak{K}$ is separable, for this suffices for the applications to field theory, and the method of proof is essentially the same in the inseparable case, except that it is necessary to use a generalization of the notion of distributions, in which the gage on the ring of the generalized distribution is not necessarily finite on the identity operator; the complications that arise may be resolved by the methods well-known in the theory of the structure of abelian rings of operators.

TheOREM 5. Let $m$ be a quasi-invariant abelian distribution defined everywhere on a (real) Hilbert space $\mathfrak{H C}$. For any vector $y$ in $\mathfrak{H C}$, let $\phi_{y}$ denote the operation of translation through $y$, and set $D_{y}=d m_{y} / d m$, where $m_{y}$ is the translation of $m$ through $y\left(m_{y}(x)=m(x)-(x, y) I\right)$. Let $U_{0}(x)$ and $V_{0}(x)$ be the transformations on $L_{2}(\mathfrak{H C}, m)$ defined as follows:

$$
U_{0}(x) f=\exp (i m(x)) f, \quad V_{0}(y) f=\phi_{y}(f)\left[\phi_{y}^{-1}\left(D_{y}\right)\right]^{-(1 / 2)}
$$

( $f$ arbitrary in $L_{2}(\mathcal{H}, m) ; x$ and $y$ arbitrary in $\mathfrak{H}$ ). Let $\mathfrak{K}$ be a complex Hilbert space of dimension $\gamma$, let $U(x)$ and $V_{1}(y)$ denote the transformations on $L_{2}(\mathcal{H}, m)$ $\times \mathfrak{K}$ that are the respective direct products of $U_{0}(x)$ and $V_{0}(y)$ with the identity on $\mathcal{K}$, and let $\mathbb{Q}$ denote the ring generated by the $U(x)$.

Then if $W(\cdot)$ is a continuous multiplier on $G$ for the representation $V_{1}(\cdot)$ relative to the ring $Q$, and if $V(\cdot)=V_{1}(\cdot) W(\cdot)$, the pair $(U(\cdot), V(\cdot))$ is a BoseEinstein canonical system over $\mathfrak{H}$. Conversely, the most general such system with separable representation space is within unitary equivalence, a discrete direct sum of systems of this type, one for each dimension $\gamma$; and for each $\gamma$ the constituent subsystem is unique, within absolute continuity of the defining distribution and equivalence of the multipliers relative to $A$.

Taking the direct part of the theorem first, it is clear that $U_{0}(\cdot)$ is a unitary representation of $\mathfrak{H C}$ with the stated continuity property. There is no special difficulty in verifying that $V_{0}$ is likewise a unitary representation, but its continuity requires examination. Note first that the restriction of $m$ to an arbitrary closed linear submanifold of $\mathfrak{K C}$ is quasi-invariant. Now it is wellknown that the only quasi-invariant distributions on finite-dimensional vector spaces are those equivalent to Lebesgue measure in the sense of absolute continuity. In the case of any such distribution, it is easily seen that the continuous dependence of $V_{0}(y)$ on $y$, in the strong operator topology, is equivalent to the well-known fact that $\int|f(x+y)-f(x)|^{2} d x \rightarrow 0$ as $y \rightarrow 0$ if $f$ is squareintegrable with respect to Lebesgue measure over the vector space, with element $d x$.

Now if $f$ is a tame operator, i.e. an element of the ring of the restriction of $m$ to a finite-dimensional submanifold, say $\Re$, the continuity of $V(y) f$ as a function of $y$ with values in $L_{2}(\mathfrak{H}, m)$, as $y$ ranges over the finite-dimensional submanifold $\mathfrak{T}$, reduces to a corresponding question in the finite-dimensional space $\Re+\mathscr{T}$, which in view of the preceding has an affirmative answer. It 
results that $V_{0}(y) f$ is a continuous function of $y$ relative to any fixed finitedimensional subspace $\mathfrak{T}$, for each of a dense set of elements $f$ of $L_{2}(\mathcal{H}, m)$. Because of the unitary character of $V_{0}(y), V_{0}(y) f^{\prime}$ is for arbitrary $f^{\prime}$ in $L_{2}(H, m)$ the uniform limit of $V_{0}(y) f$ with $f$ a tame operator and hence $V_{0}(y) f^{\prime}$ depends continuously on $y$ when $y$ varies over a finite-dimensional subspace. The remainder of the proof of the direct part of the theorem is a straightforward adaptation of standard techniques and the details may be omitted.

Conversely, let $(U(\cdot), V(\cdot))$ be a given Bose-Einstein canonical system over $\mathcal{H}$ with separable representation space $\mathfrak{K}$. The relation, $U(x) V(y) U(-x)$ - $V(-y)=e^{i(x, y)} I$, shows that the inner automorphism of the ring of all bounded operators on $\mathcal{K}$ that is induced by $V(y)$ leaves invariant the abelian ring $B$ generated by the $U(x)$. By the structure theory for abelian rings of operators [9], there exist unique mutually orthogonal projections $P_{\gamma}$ in $B(\gamma=1,2, \cdots, \infty)$ which commute with the $V(y)$, whose ranges span $\mathcal{K}$, and such that the restriction of $B$ to the range of $P_{\gamma}$ is, within unitary equivalence, the set of all direct products of operators in a maximal abelian ring $a_{\gamma}$ with the identity on a complex Hilbert space of dimension $\gamma$. Because of the separability of $\Re$, there exists a (nonsingular) probability gage $E_{\gamma}$ on $a_{\gamma}$, and defining $m_{\gamma}(x)=Q_{\gamma}(x)$, where $Q_{\gamma}(x)$ is the self-adjoint operator on the range of $P_{\gamma}$ such that $\exp \left(i t Q_{\gamma}(x)\right)=U_{\gamma}(t x),-\infty<t<\infty$, where $U_{\gamma}(\cdot)$ denotes the representation of the additive group of $\mathcal{F}$ obtained by contraction of $U(\cdot)$ to the invariant subspace consisting of the range of $P_{\gamma}$, it is clear that $m_{\gamma}$ is a distribution on $\mathfrak{H C}$ (relative to the gage $E_{\gamma}$ ). This distribution is quasiinvariant because the commutation relations imply that $V_{\gamma}(-y) m_{\gamma}(x) V_{\gamma}(y)$ $=m_{\gamma}(x)-(x, y) I_{\gamma}$, showing in fact that $V_{\gamma}(y)$ itself induces the automorphism of translation through $y$. From these observations the remainder of the converse part follows by familiar methods.

The canonical system $\left(U_{0}, V_{0}\right)$, which evidently plays a fundamental role in the foregoing, may be called the system determined by the quasi-invariant distribution $m$ on $\mathfrak{F}$. In the finite case any such system is irreducible. The following result shows that this is the case somewhat more generally (although it seems doubtful whether it holds for arbitrary quasi-invariant distributions), and in particular holds for the canonical normal distribution on any Hilbert space, in which case the result is essentially equivalent, via the duality transform, to a result of Cook [Trans. Amer. Math. Soc. vol. 74 (1953) pp. 222245, Theorem 7]. Another form of the results asserts that vector translations act ergodically on a Hilbert space, relative to any continuous quasi-invariant distribution admitting a spanning set of finite-dimensional stochastically independent manifolds.

COROLlary 5.0. The canonical system determined by a quasi-invariant distribution on a Hilbert space $\mathfrak{H C}$ is irreducible in case $\mathfrak{H C}$ is the direct sum of stochastically independent finite-dimensional manifolds. 
To prove this, note that the $U(x)$ generate the same ring $R$ as that determined by the $m(x)$, which is maximal abelian, being both standard and abelian. Hence any bounded linear operator $T$ on $L_{2}(\mathfrak{H}, m)$ that commutes with both the $U(x)$ and $V(y)$ is an element of the ring of $m$ that commutes with all $V(y)$. Hence for any operator $S$ in $R, V(y) T S=T V(y) S$, or $\phi_{y}(T)$ $\cdot \phi_{y}(S) C=T \phi_{y}(S) C$, where $C$ is the operator $\left[\phi_{y}^{-1}\left(D_{y}\right)\right]^{-(1 / 2)}$. As $C$ is invertible, it results that $\phi_{y}(T)=T$ for arbitrary $y$ in $\mathfrak{K}$.

To conclude the proof of irreducibility, it therefore suffices to show that the only operators $T$ in $R$ such that $\phi_{y}(T)=T$ for all $y$ are scalar multiples of the identity. To this end let $P_{M}$ denote the operation of projecting $\mathcal{F}$ onto the subspace $\mathfrak{T}$, and let $P_{M}^{\prime}$ denote the operation of projecting $L_{2}(\mathfrak{H C}, m)$ onto the subspace $L_{2}(\mathfrak{T}, m)$ of operators based on $\mathfrak{T}$ (i.e. affiliated with the ring determined by the $m(x)$ with $x$ in $\mathfrak{T})$. Then $P_{M}^{\prime} \rightarrow I$ strongly as $\mathfrak{T}$ ranges over the directed system of finite direct sums of the given stochastically independent subspaces, since these subspaces span and $m$ is continuous (cf. the proof of Theorem 2). Further, for any such subspace $\mathfrak{T}, P_{M}^{\prime} \phi_{y}(S)=\phi_{y}\left(P_{M}^{\prime} S\right)$ if $y$ is in $\mathfrak{M}$, for arbitrary $S$ in $\mathbb{R}$.

For $P_{M}^{\prime} X$ is for any $X$ in $R$ the conditional expectation of $X$ with respect to the subring $R_{M}$ of $R$ determined by the restriction of $m$ to $\mathscr{T}$. To see this it suffices, since $P_{M}^{\prime} X$ is clearly affiliated with $R_{M}$, and is integrable as it is in $L_{2}(\mathfrak{F C}, m)$, to show that $E\left[P_{M}^{\prime}(X) Y\right]=E[X Y]$ for all self-adjoint $Y$ in $\Omega_{M}$. This is clear from the fact that $P_{M}^{\prime}$ is a projection on $L_{2}(\mathfrak{H C}, m)$, so that $E\left[P_{M}^{\prime}(X) Y\right]=E\left[X P_{M}^{\prime}(Y)\right]$, which equals $E[X Y]$ because obviously $P_{M}^{\prime} Y$ $=Y$. Now the operation of taking conditional expectation is easily seen to be a contraction on $L_{\infty},\left|E\left[X \mid R_{M}\right]\right|_{\infty} \leqq|x|_{\infty}$, by an argument similar to that used to show it is a contraction on $L_{1}$. Note also that $\phi_{y}$ takes $R_{M}$ into itself, for it takes $m(x)(x$ in $\mathfrak{T})$ into $m(x)-(x, y) I$, so that $\phi_{y}\left(P_{M}^{\prime} S\right)$ is in $\Omega_{M}$. As obviously $P_{M}^{\prime} \phi_{y}(S)$ is in $R_{M}$, to prove the stated equality it suffices to show that $E\left[P_{M}^{\prime}\left(\phi_{y}(S)\right) X\right]=E\left[\phi_{y}\left(P_{M}^{\prime} S\right) X\right]$ for all self-adjoint $X$ in $\mathcal{R}_{M}$. By definition of the conditional expectation, $E\left[P_{M}^{\prime}\left(\phi_{y}(S) X\right]=E\left[\phi_{y}(S) X\right]\right.$, which in turn equals $E\left[S \phi_{y}^{-1}(X) D_{y}\right]$. On the other hand,

$$
E\left[\phi_{y}\left(P_{M}^{\prime} S\right) X\right]=E\left[\left(P_{M}^{\prime} S\right) \phi_{y}^{-1}(X) D_{y}\right]=E\left[E\left\{\left(P_{M}^{\prime} S\right) D_{y} \mid \Omega_{M}\right\} \phi_{y}^{-1}(X)\right]
$$

(as $\phi_{y}^{-1}(X)$ is in $\left.\Omega_{M}\right)=E\left[P_{M}^{\prime}(S) E\left\{D_{y} \mid \Omega_{M}\right\} \phi_{y}^{-1}(X)\right]$ (as the operation of taking the conditional expectation is multiplicative when one factor is affiliated with the subring) $=E\left[S \phi_{y}^{-1}(X) E\left\{D_{y} \mid \mathscr{R}_{M}\right\}\right]$ (by the definition of conditional expectation). Hence the stated equality holds if (and only if) $E\left[D_{y} \mid R_{M}\right]=D_{y}$, which equation follows directly from the circumstances that $M$ and its orthocomplement are stochastically independent and that $y$ is in $M$.

2. Transformations on canonical systems. The transformation of one formal Bose-Einstein canonical set into another: $Q_{j} \rightarrow Q_{j}^{\prime}, \quad P_{k} \rightarrow P_{k}^{\prime}$ $(j, k=1,2, \cdots)$ is used frequently in the physical literature, and may be called a pseudo-canonical transformation, in distinction from the strictly 
canonical type that are induced by a unitary transformation. The latter type of transformation does not alter the spectra of operators, but a pseudocanonical transformation may well carry a well-defined self-adjoint operator into an "infinite" one with vacuous domain. (This may be shown by considering the effect of the pseudo-canonical transformation $Q_{j} \rightarrow a_{j} Q_{j}, P_{k} \rightarrow a_{k}^{-1} P_{k}$, on a linear form in the $Q_{j}$, a type of operator fairly typical of realistic interaction operators in field theory.)

The purpose of the present section is to illuminate the disparity between pseudo- and strictly canonical transformations by showing that pseudocanonical transformations of a simple sort are generally not strictly canonical, and by obtaining a necessary and sufficient condition for strict canonicity. It is convenient to introduce the notion of a basic infinitesimal canonical system, which we define as the restrictions to a basis for the underlying Hilbert space $\mathcal{H}$ of an infinitesimal canonical system $P(\cdot), Q(\cdot)$, over $\mathcal{H}$. For simplicity of statement we shall treat only the case of principal interest, in which $\mathfrak{F C}$ is of denumerable dimension (the extension to the general case being immediate). In this case a basic set may be written as a pair of sequences $P_{1}, P_{2}, \cdots$ and $Q_{1}, Q_{2}, \cdots$ of self-adjoint operators, where $P_{j}=P\left(e_{j}\right)$ and $Q_{k}=Q\left(e_{k}\right)$, and $e_{1}, e_{2}, \cdots$ is an orthonormal basis for $\mathfrak{H}$.

Corollary 5.1. Let $P_{1}, P_{2}, \cdots$ and $Q_{1}, Q_{2}, \cdots$ be the infinitesimal canon$i$ cal system over a Hilbert space $\mathfrak{H}$, relative to the orthonormal basis $e_{1}, e_{2}, \cdots$ of $\mathrm{F}$, which are stochastically independent with respect to the quasi-invariant abelian distribution $m$ on $\mathfrak{K C}$, which determines the given canonical system. Let $\phi_{1}, \phi_{2}, \ldots$ be a sequence of continuously differentiable real-valued functions on the real line with $\phi_{k}^{\prime}(x)>0$ for all $x$ and $k$.

Then in order that there exist a unitary transformation $U$ such that $U^{*} Q_{k} U$ $=\phi_{k}\left(Q_{k}\right)(k=1,2, \cdots)$, it is necessary and sufficient that the following product be convergent:

$$
\prod_{k} \int_{-\infty}^{\infty}\left[p_{k}(t) p_{k}\left(\phi_{k}(t)\right) \phi_{k}^{\prime}(t)\right]^{(1 / 2)} d t
$$

where $p_{k}$ is the probability density of the distribution of the operator $m\left(e_{k}\right)$. In the event that such a unitary operator exists, it may be chosen so that $U^{*} P_{k} U$ is affiliated with the ring determined by $P_{k}$ and $Q_{k}(k=1,2, \cdots)$.

If there exists a unitary operator $U$ such that $U^{*} Q_{k} U=\phi_{k}\left(Q_{k}\right)\left(=Q_{k}^{\prime}\right.$ say), for all $k$, let $m^{\prime}(x)=U^{*} m(x) U$. Then $m^{\prime}$ is absolutely continuous with respect to $m$, so that by Theorem 2 , the product $\prod_{k}\left[d m_{k}^{\prime} / d m_{k}\right]^{(1 / 2)}$ must be convergent. (Note that the $e_{k}$ are stochastically independent with respect to $m^{\prime}$ as well as $m$.) A simple computation shows that this is termwise equal to the given product.

Now suppose the given product is convergent. Let $\mathfrak{K}_{n}=L_{2}\left(\mathcal{H}_{n}, m_{n}\right)$, where $\mathcal{F}_{n}$ is the one-dimensional subspace spanned by $e_{n}(n=1,2, \cdots)$ and 
$m_{n}$ is the restriction of $m$ to $\mathcal{H}_{n}$. Then $\mathscr{K}_{n}$ is unitarily equivalent to the space $L_{2}\left(-\infty, \infty ; p_{n}\right)$ of all square-integrable complex-valued functions on the line, relative to the probability density $p_{n}$ giving the distribution of $m\left(e_{n}\right)$, in such a way that $Q_{k}$ corresponds to multiplication by the function $x$. (Note that the distribution of $m\left(e_{k}\right)$ is absolutely continuous because of the quasi-invariance of $m$.) Let $W_{n}$ denote the unitary operator on $L_{2}\left(-\infty, \infty ; p_{n}\right): f(t)$ $\rightarrow f\left(\phi_{n}^{-1}(t)\right)\left[p_{n}\left(\phi_{n}^{-1}(t)\right) \phi_{n}^{\prime}\left(\phi_{n}^{-1}(t)\right) p_{n}(t)\right]^{(1 / 2)}\left(f\right.$ arbitrary in $L_{2}\left(-\infty, \infty ; p_{n}\right)$ and let $W_{n}^{\prime}$ be the corresponding unitary operator on $\mathfrak{K}_{n}$, via the unitary equivalence described (which is not unique, but a fixed one is chosen for each $n$ and used thereafter). This induces an automorphism $A_{n}: X \rightarrow W_{n}^{\prime *} X W_{n}^{\prime}$ on the ring of all bounded linear operators on $\mathscr{K}_{n}$. Now consider the condition of Theorem 5 , with reference to the formulation of $\left(L_{2}(\mathfrak{H}, m), I\right)$ as the product of the $\left(\mathcal{K}_{n}, I\right)$ that was noted in connection with that theorem. It is easy to see that the $\lambda$ th factor is $\left|\left(W_{\lambda} z_{\lambda}, z_{\lambda}\right)\right|^{2}$, if $W_{\lambda}$ is a unitary operator implementing the automorphism $A_{\lambda}$. Using the given form for $W_{n}$ and noting that the unitary equivalence taking $W_{n}^{\prime}$ into $W_{n}$ takes $I$ into the function that is identically one on $(-\infty, \infty)$, the factor $\left(W_{n}^{\prime} z_{n}, z_{n}\right)$ is readily evaluated as identical with the $n$th factor in the product given in Corollary 5.1.

Thus there exists an automorphism $A$ extending all the $A_{n}$ in the fashion indicated in Theorem 5. This automorphism has the form $X \rightarrow U^{*} X U$ for some unitary operator $U$, whose restriction to $L_{2}\left(\mathcal{H}_{n}, m_{n}\right)$ extends the transformation induced by $W_{n}^{\prime}$. It is clear that this takes $Q_{n}$ into $Q_{n}^{\prime}$ and takes $P_{n}$ into some self-adjoint operator on $L_{2}\left(\mathcal{F}_{n}, m_{n}\right)$, and any such operator is affiliated with the ring determined by $P_{n}$ and $Q_{n}$ because the system determined by a distribution in one dimension is irreducible.

In the foregoing result the $Q_{n}^{\prime}$ were specified, while the $P_{n}^{\prime}$ were not completely specified. The following result deals with the transformation question for the case when the $P_{n}^{\prime}$ are specified as well.

Corollary 5.2. Let $P_{1}, P_{2}, \cdots$ and $Q_{1}, Q_{2}, \cdots$ be as in Corollary 5.1, and let $P_{1}^{\prime}, P_{2}^{\prime}, \cdots$ and $Q_{1}^{\prime}, Q_{2}^{\prime}, \cdots$ be a second infinitesimal Bose-Einstein canonical system over $\mathfrak{H C}$, relative to the same base and on the same representation space, and such that $P_{n}^{\prime}$ and $Q_{n}^{\prime}$ are affiliated with the ring determined by $P_{n}$ and $Q_{n}$. A necessary and sufficient condition that there exist a unitary transformation simultaneously transforming the $P_{i}$ and $Q_{j}$ into the $P_{i}^{\prime}$ and $Q_{j}^{\prime}$ $(i, j=1,2, \cdots)$ is that: (1) for each $n$ there exist a unitary transformation $U_{n}$ transforming $P_{n}$ and $Q_{n}$ simultaneously into $P_{n}^{\prime}$ and $Q_{n}^{\prime}$ respectively, and affiliated with the ring determined by $P_{n}$ and $Q_{n}$; (2) the product $\prod_{k}\left|\int_{-\infty}^{\infty} u_{k}(t) p_{k}(t) d t\right|$ be convergent, where $p_{k}$ is as in Corollary 5.1 and $u_{n}$ is the transform of the function identically one on $(-\infty, \infty)$ under the transform of $U_{n}$ by a unitary equivalence of $L_{2}\left(\mathcal{F C}_{n}, m_{n}\right)$ with $L_{2}\left(-\infty, \infty ; p_{n}\right)$ (that takes $Q_{n}$ into multiplication by $x$ and $P_{n}$ into the self-adjoint generator of translation).

Since $P_{n}$ and $Q_{n}$ jointly act irreducibly on $L_{2}\left(\mathcal{H}_{n}, m_{n}\right)$, any automorphism 
of all bounded operators on this space is determined by its extended action on $P_{n}$ and $Q_{n}$. Similarly $P_{1}, P_{2}, \cdots$ and $Q_{1}, Q_{2}, \cdots$ form an irreducible set on $L_{2}(\mathfrak{C}, m)$ and so any automorphism of all bounded operators on this space is determined by its action on the $P$ 's and $Q$ 's. Thus there exists a unitary transformation making the indicated transformation between the two canonical systems if and only if there exists an automorphism $A$ as in Theorem 5, i.e. if and only if the product given there is convergent.

As illustrations of the use of the foregoing criteria and for their intrinsic interest we consider some examples. In all cases considered the distribution $m$ will be taken to be the canonical normal distribution, as the corresponding canonical system is that which is implicitly designated in the standard theory, -in so far as there exists any designation beyond the commutation relations (cf. Dirac [3]),--and is mathematically distinguished as well; and it would be easy to treat more general cases along the same lines $\left({ }^{4 a}\right)$.

EXAMPLE 1. When may the transformation $Q_{n} \rightarrow a_{n} Q_{n}\left(a_{n}>0\right)$ be extended to a strictly canonical transformation? Applying Corollary 5.1, there is a unitary transformation implementing the transformation if and only if

$$
\prod_{k} \int\left[\exp \left(-t^{2} / 2 c\right) \exp \left(-a_{k}^{2} t^{2} / 2 c\right) a_{k}\right]^{(1 / 2)}(2 \pi c){ }^{-(1 / 2)} d t
$$

is a convergent product, where $c$ denotes the variance parameter of the distribution. Evaluating the integrals, it is found that this is equivalent to the convergence of $\prod_{k}\left(2 a_{k} /\left(1+a_{k}^{2}\right)\right)$, showing that it is necessary (but not sufficient) that $a_{k} \rightarrow 1$. An equivalent form of the condition, which may be obtained by standard estimates, is that $\sum_{k}\left(a_{k}-1\right)^{2}$ be convergent. In the event of convergence, $P_{n}$ may be transformed into $a_{n}^{-1} P_{n}$ simultaneously with the transformation of the $Q_{n}$ in to the $a_{n} Q_{n}$.

EXAMPLE 2. When is the transformation $Q_{j} \rightarrow Q_{j}, P_{k} \rightarrow P_{k}+a_{k} I$, strictly canonical? Here the transform of $U_{n}$ occurring in Corollary 5.2 is the operation $f(t) \rightarrow e^{i a_{n} t} f(t)$, so the existence of a unitary effecting the given transformation is equivalent to the convergence of the product

$$
\mathrm{II}_{k} \int e^{i a_{n} t} \exp \left(-t^{2} / 2 c\right)(2 \pi c)^{-(1 / 2)} d t
$$

which leads readily to the condition that $\sum_{n} a_{n}^{2}$ be convergent. In particular, the transformation $Q_{j} \rightarrow Q_{j}, P_{k} \rightarrow P_{k}+I(j, k=1,2, \cdots)$ is not strictly canon-

$(4 \mathrm{a})$ Indications of the existence of inequivalent representations are given in [4] and [5]. In [4] systems analogous to those of Example 2 are cited; and an argument is given (pp. 149151) indicating that there exists a system $\left\{P_{j}, Q_{k}\right\}$ of densely defined operators on Hilbert space such that $P_{j} Q_{k}-Q_{k} P_{j}=i \delta_{j k}$, and which is unitarily inequivalent to the system $\left\{P_{j}\right.$, $\left.Q_{k}+a_{k} I\right\}$, when $\sum_{k} a_{k}^{2}=\infty$. In [5] systems are cited corresponding to those of Example 1 for the special case when the $a_{k}$ are bounded away from unity; the proof of inequivalence is not indicated. 
ical, showing the existence of nontrivial multipliers for the case of an irreducible canonical system arising from the canonical normal distribution, and in fact of orthogonally invariant ones (i.e. such as are independent of the choice of basis in $H$ ).

EXAmple 3. When is the transformation $Q_{j} \rightarrow Q_{j}, P_{k} \rightarrow P_{k}+\sigma_{k} Q_{k}$ strictly canonical? Mathematically the latter operators are not manifesty self-adjoint, so it is more satisfactory to define them as the transforms of $P_{k}$ under the unitary transformation on $L_{2}\left(\mathcal{F}_{k}, m_{k}\right)$ corresponding to the transformation on the line: $f(x) \rightarrow \exp \left(i x^{2} \sigma_{k} / 2\right) f(x)$. A computation analogous to that in Example 2 leads to the condition that $\sum_{n} \sigma_{n}^{2}$ be convergent.

Example 4. The method used to obtain Corollaries 5.1 and 5.2 applies more generally to e.g. the case when $\mathfrak{H C}$ is the direct sum of stochastically independent finite-dimensional submanifolds. An example of this shows, in very rough physical terms, that there exists no unitary transformation taking a pair of identical uncoupled bosons into the corresponding pair of bosons coupled in bilinear fashion. Mathematically this may be formulated as follows. $\mathfrak{F}=\mathfrak{C}^{(1)}+\mathfrak{H C}^{(-1)}$, where there is a distinguished isomorphism between $\mathcal{F}^{(1)}$ and $\mathfrak{F}^{(-1)}$ taking the orthonormal basis $\left\{e_{j}^{(1)}\right\}$ of $\mathscr{F}^{(1)}(j=1,2, \cdots)$ into the orthonormal basis $\left\{e_{j}^{(-1)}\right\}$ of $\mathfrak{F}^{(-1)}$. The basic infinitesimal canonical operators may be correspondingly labeled as $P_{j}^{(\alpha)}$ and $Q_{j}^{(\alpha)}(\alpha= \pm 1 ; j=1,2, \cdots)$. Now consider the pseudo-canonical transformation $Q_{j}^{(\alpha)} \rightarrow Q_{j}^{(\alpha)}, P_{j}^{(\alpha)} \rightarrow P_{j}^{(\alpha)}$ $+Q_{j}^{(-\alpha)}$; as in Example 3, the latter operator is to be strictly defined as the transform of $P_{j}^{(\alpha)}$ under the unitary transformation on $L_{2}\left(\mathfrak{H}_{j}, m_{j}\right)$, where $\mathfrak{H}_{j}$ is the two-dimensional subspace of $\mathfrak{H C}$ spanned by the $e_{j}^{(\alpha)}(\alpha= \pm 1)$, corresponding to the transformation of functions on the plane, $f(x, y) \rightarrow e^{i x y} f(x, y)$. The stochastically independent subspaces $\mathfrak{H}_{j}$ are here two-dimensional, which is clearly immaterial for the proof of Corollary 5.2, and the implementability of the given transformation by a unitary is equivalent to the convergence of the product $\prod_{k} \tau_{k}$, with $\tau_{k}=\iint e^{i x y} \exp \left[-\left(x^{2}+y^{2}\right) / 2 c\right](2 \pi c)^{-1} d x d y$. As $\tau_{k} \neq 1$, the product diverges.

\section{Fermi-Dirac CANONICAL Systems}

1. Covariant systems with a trace. It is difficult to achieve significant results in this case without making fuller use of the physical desiderata than was necessary in the Bose-Einstein case. In field theory one is concerned not with arbitrary canonical systems, but in principle only with those that are covariant and admit a trace. More precisely, a system $\{P(\cdot), Q(\cdot)\}$, over $\mathfrak{H}$, may be said to be covariant with respect to a representation $U$ of a group $G$ by orthogonal transformations on $\mathcal{H C}$ and a unitary representation $\Gamma$ of $G$ on the representation space $\nVdash$ on which the system operators act, in case

$$
P(U(a) x)=\Gamma(a)^{-1} P(x) \Gamma(a) \text { and } Q(U(a) x)=\Gamma(a)^{-1} Q(x) \Gamma(a),
$$

for arbitrary $a$ in $G$ and $x$ in $\mathfrak{F}$. The system may be said to admit a trace in case there exists a linear functional $E \neq 0$ on the ring determined by the $Q(x)$, 
that is positive: $E(A * A) \geqq 0$ for any bounded operator $A$ in the ring, and central: $E(A * A)=E(A A *)$ always. The manner indicated earlier of going from free to interacting fields preserves these features, which are present in the free field case based on the Clifford distribution [12].

In the present section we treat only the simple case when $G$ is the group of all orthogonal transformations on $\mathfrak{H}$, and $U$ and $\Gamma$ are the corresponding natural representations. Specifically, $U(a)=a$, while $\mathcal{K}$ is the space of all square-integrable operators on $\mathfrak{H C}$ (relative to the Clifford distribution), on which $G$ acts canonically to give the representation $\Gamma$ of $G$. It will be clear that the proof applies to a certain apparently fairly substantial class of subgroups of the orthogonal group (quite possibly including all images of complex semisimple Lie groups under irreducible representations).

The basic canonical system here may be called the "free-field" system (cf. $[12])\left({ }^{5}\right)$, and for present purposes may be defined as follows. Let $K_{0}$ be the (essentially unique) algebra over the complex field with an adjunction operation $*$, that contains and is generated (as an algebra) by the real Hilbert space $\mathfrak{F}$ together with a unit $e_{0}$, bears a probability gage $E$, and is such that $x y+y x=(x, y) e_{0}$ for arbitrary $x$ and $y$ in $\mathfrak{H}$. Let $\mathcal{K}$ be the completion of $\mathscr{K}_{0}$ with respect to the inner product $(u, v)=E(v * u)$, for arbitrary $u$ and $v$ in $\varkappa_{0}$, and let multiplication be defined between an element of $\varkappa_{0}$ and an element of $\mathcal{K}$ by continuous extension of the multiplication in $\mathcal{K}_{0}$. The freefield system $(P, Q)$ over $\mathfrak{H}$ is then given by the equations

$$
P(x) u=i \Omega u \cdot x 2^{-(1 / 2)}, \quad Q(x) u=2^{-(1 / 2)} x \cdot u,
$$

where $\Omega$ is the continuous extension to $\mathscr{K}$ of the automorphism of $\mathcal{K}_{0}$ induced by the transformation $x \rightarrow-x$ on $\mathfrak{H}$. A closely related system $\left(P^{\prime}, Q^{\prime}\right)$ with $P^{\prime}(x)=-P(x), Q^{\prime}(x)=Q(x)$, might be called the anti-free-field system, for from a physical outlook it differs from the foregoing system only in having negative rather than positive occupation numbers. It is not unitarily equivalent to the free-field system, when $\mathfrak{H}$ is infinite-dimensional, but may be obtained from it by transforming by a conjugation (or involutory anti-unitary operator) $\left({ }^{6}\right)$.

THEOREM 6. Let $P(\cdot)$ and $Q(\cdot)$ be linear maps from the real infinite-dimensional Hilbert space $\mathfrak{H C}$ to the self-adjoint operators on the Hilbert space $\mathfrak{K}$ of all square-integrable operators with respect to the Clifford distribution on $\mathcal{H}$. Suppose that:
(1) $P(U x)=\Gamma(U)^{-1} P(x) \Gamma(U)$
and
$Q(U x)=\Gamma(U)^{-1} Q(x) \Gamma(U)$, where for

(5) On p. 174 of this reference, the equation $C_{x}=(P(x)+i Q(x)) 2^{(1 / 2)}$ should be replaced by the equation $C_{x}=(P(x)+i Q(x)) 2^{-(1 / 2)}$ to obtain a correct definition of the $P(x)$ and $Q(x)$.

$\left.{ }^{(}\right)$Examples of inequivalent Fermi-Dirac canonical systems were known to J.von Neumann (according to verbal communication from him), and in fact it follows from a result cited in [16, p. 77], that such a system may generate a ring of type $\mathrm{II}_{\infty}$. However, such a system can not be obtained by transformation of the free-field system, and seems likely to be physically inappropriate. 
any orthogonal operator $U$ on $\mathfrak{H}, \Gamma(U)$ is the induced operator on $\mathfrak{K}$;

(2) there exists a trace on the ring determined by the $Q(x)$;

(3) $(P(\cdot), Q(\cdot))$ form a Fermi-Dirac canonical system over $\mathcal{H}($ i.e. $Q(x) Q(y)$ $+Q(y) Q(x)=(x, y) I=P(x) P(y)+P(y) P(x)$ and $Q(x) P(y)+P(y) Q(x)=0$ for arbitrary $x$ and $y$ in $\mathfrak{F C})$.

Then the system $(P(\cdot), Q(\cdot))$ is unitarily equivalent either to the free-field canonical system or to its transform under a conjugation.

It is no essential loss of generality to normalize the given trace function $E$ on the ring $Q$ generated by the $Q(x)$ by requiring $E(I)=1$.

For any finite-dimensional subspace $\mathbb{M}$ of $\mathfrak{H C}$, the $Q(x)$ for $x$ in $\mathbb{M}$ generate a subalgebra $Q_{M}$ of the ring $Q$ determined by the $Q(x), x$ ranging over $\mathcal{H}$, that is isomorphic to the Clifford algebra over $\mathfrak{M}$. Now when $\mathfrak{T}$ has even dimension, it is isomorphic to a total matrix algebra, on which there is clearly a unique function $E_{M}$ having the properties of the contraction to $A_{M}$ of $E$,- namely, the normalized trace. Therefore $E$ agrees on the finite products of the $Q(x)$ with the expectation relative to the Clifford distribution; and these span a dense subset of $A$, so that $E$ must agree with the expectation functional for the Clifford distribution on $\mathfrak{H}$.

Thus it may be assumed that $Q(x)=Q_{0}(x)$. Now set

$$
W(x)=\left[P(x)-P_{0}(x)\right] \Omega,
$$

where $\Omega$ is the automorphism induced by the transformation $x \rightarrow-x$, on $L_{2}(\mathcal{F}, m)$. Then $W(x)$ commutes with all $Q(y), y$ in $\mathcal{H}$. Now as $m$ is a standard representative, $Q$ is the left ring of a Hilbert algebra $\mathcal{K}_{0}$ (which may be taken to include all bounded elements), so that any bounded linear operator commuting with any set of generators for it must be a right multiplication by a bounded element of the Hilbert algebra. Hence $W(x)$ is the operation of right multiplication by an element $w(x)$ of $\mathfrak{K}_{0}$, and as this element is unique, $w(\cdot)$ is linear on $\mathfrak{K C}$ to $\mathfrak{K}_{0}$. By covariance, $\Gamma(U) w(x)=w(U x)$, for any orthogonal transformation $U$ on $H$. We show next that this implies that $w(x)=\alpha q(x)$, for some constant $\alpha$, where $q(x)$ is that element of the Hilbert algebra such that $Q(x)$ is the operation of left multiplication by $q(x)$.

To this end, let $w^{\prime}$ and $\Gamma^{\prime}$ denote the transforms of $w$ and $\Gamma$ under the duality transform [12]. The relation $\Gamma^{\prime}(U) w^{\prime}(x)=w^{\prime}(U x)$ then holds, and as $\Gamma^{\prime}(U)$ commutes with the operations of projection on the subspaces of all skew-symmetric $r$-tensors $(r=0,1,2, \cdots)$, it is no essential loss of generality to assume that $w^{\prime}(x)$ is for all $x$ a pure $r$-tensor. The case $r=0$ is ruled out by the linearity of $w$, so we need only show that it is impossible that $r>1$. Now $w^{\prime}$ is a linear transformation from $\mathfrak{F}$ into the Hilbert space $\mathfrak{K}^{(r)}$ of skewsymmetric $r$-tensors over the complex extension of $\mathfrak{H}$, and the above equation states that it connects the identical representation $U \rightarrow U$ of the orthogonal group on $\mathfrak{F}$ with a subrepresentation of that induced in the skew-symmetric 
$r$-tensors. This connection holds in particular for the subgroup of the orthogonal group leaving invariant the orthocomplement of any finite-dimensional subspace of $\Re$. But the known form of the action of the orthogonal group on tensors (see e.g. H. Weyl, The classical groups, Princeton, 1939) precludes an inclusion between the representation induced in the skew-symmetric $r$ tensors $(r>1)$ and the given group (as acting on vectors), if the dimension of the vector space exceeds $r$. As this vector space may here be taken to be of arbitrarily high dimensions, it follows that $r=1$.

Thus $P(x)=P_{0}(x)+\alpha R_{q(x)} \Omega$, where $R_{q(x)}$ is the operation of right multiplication by $q(x),=\gamma P_{0}(x)$ for some constant $\gamma$. From the requirement that $P(x)^{2}=P_{0}(x)^{2}=I / 2$, it results that $\gamma= \pm 1$, yielding the free-field and antifree-field canonical systems.

It is easily seen that these two systems are equivalent under a conjugation, in fact the duality transform of the canonical conjugation on the space of covariant skew-symmetric tensors over the complex extension of $\mathfrak{H C}$ is a conjugation effecting the equivalence. To show that the two systems are not unitarily equivalent, assume on the contrary that there exists a unitary operation $T$ effecting such an equivalence: $T^{-1} Q(x) T=Q(x)$ and $T^{-1} P(x) T$ $=-P(x)$, for all $x$ in $\mathfrak{F}$. Then $T$ is in the commutor of the ring generated by the $Q(x)$, and so by the known form for the commutor of a standard ring, must be a right multiplication by some element $t$ of the Hilbert ring of the Clifford distribution.

The transformation properties of the $P(x)$ under $T$ then signify that $\Omega t \cdot x=-x \cdot t$, for all $x$ in $\mathcal{H}$. Taking adjoints and noting that $t *=t^{-1}$, it follows that also $x \cdot \Omega t=-t \cdot x$. It results that $\Omega t-t$ commutes with all $x$ and $\Omega t+t$ anti-commutes with all $x$. Now the ring of the Clifford distribution on an infinite-dimensional space is a factor, so that $\Omega t-t$ must be a scalar multiple of the identity, and taking expectations, it follows that $\Omega t-t=0$. Thus it suffices to show that $t x=-x t$ can hold for all $x$ only when $t=0$. Now all elements $t$ of the Hilbert space that is the completion of the Hilbert algebra of the Clifford distribution that satisfy these equations form a subspace that is invariant under the orthogonal group on $\mathfrak{F}$, acting in canonical fashion. Using the duality transform, and noting that the orthogonal group on $\mathfrak{H}$ acts irreducibly on the covariant skew-symmetric (square-integrable) $r$-tensors over the complex extension of $\mathscr{H C}$ (by approximation with the case of the finite-dimensional orthogonal group, along lines parallel to those developed in detail in the case of the unitary group in [11]), it follows that there is a nontrivial solution $t$ to the equations $t x=-x t, x$ arbitrary in $\mathfrak{H}$, only if there exists a rank $r$ such that the equation holds for all $t$ that are duality transforms of $r$-tensors. But if $t=e_{1} \cdot e_{2} \cdots e_{r}$, and $x=e_{1}$ or $e_{r+1}$ depending on the parity of $r$, where $e_{1}, e_{2}, \cdots, e_{r+1}$ are orthonormal in $\mathfrak{F}$, the equation fails to hold.

2. Transformations on canonical systems. This section presents a result 
on the Fermi-Dirac case that is parallel to the result in Part II on transformation of Bose-Einstein canonical systems, relative to a given orthonormal basis. A Fermi-Dirac canonical system over $\mathcal{H}$ may be defined as an ordered pair $(P(\cdot), Q(\cdot))$ of linear maps from $\mathfrak{H C}$ to the bounded self-adjoint linear operators on a complex Hilbert space $\kappa$, satisfying condition $(3)$ of Theorem 7. The two sequences $\left\{P\left(e_{j}\right)\right\},\left\{Q\left(e_{j}\right)\right\}(j=1,2, \cdots)$, where $e_{1}, e_{2}, \cdots$ is an orthonormal basis for $\mathfrak{H C}$, may be called a canonical system relative to the basis $e_{1}, e_{2}, \cdots$. The following corollary treats only the case of principal interest, but its method applies somewhat more generally to systems admitting a trace.

Corollary 5.3. Let $P_{1}, P_{2}, \cdots$ and $Q_{1}, Q_{2}, \cdots$ be the Fermi-Dirac freefield canonical system over the real Hilbert space $\mathfrak{H C}$, with respect to an orthonormal basis $e_{1}, e_{2}, \cdots$, with representation space $\kappa$. Let $P_{1}^{\prime}, P_{2}^{\prime}, \cdots$ and $Q_{1}^{\prime}, Q_{2}^{\prime}, \cdots$ be two sequences of bounded self-adjoint operators on $\mathfrak{K}$ such that $P_{j}^{\prime} P_{k}^{\prime}+P_{k}^{\prime} P_{j}^{\prime}=I \delta_{j k}=Q_{j}^{\prime} Q_{k}^{\prime}+Q_{k}^{\prime} Q_{j}^{\prime}$, while $P_{j}^{\prime} Q_{k}^{\prime}+Q_{k}^{\prime} P_{j}^{\prime}=0$ $(j, k=1,2, \cdots)$, and with $P_{j}^{\prime}$ and $Q_{j}^{\prime}$ in the ring $Q_{j}$ generated by $P_{j}$ and $Q_{j}$. $A$ necessary and sufficient condition that there exist a unitary operator $U$ on $K$ such that $U^{*} P_{j} U=P_{j}^{\prime}$ and $U^{*} Q_{j} U=Q_{j}^{\prime}(j=1,2, \cdots)$ is that the product $\Pi_{j}\left|\left(w_{11}^{(j)}+w_{12}^{(j)}+w_{21}^{(j)}+w_{22}^{(j)}\right) / 2\right|$ be convergent, where $\left(w_{r s}^{(j)}\right)$ is a $2 \times 2$ matrix corresponding to a unitary element $V^{(j)}$ in $\Omega_{j}$ such that $V^{(j)-1} P_{j} V^{(j)}=P_{j}^{\prime}$, $V^{(j)-1} Q_{j} V^{(j)}=Q_{j}^{\prime}$, in an isomorphism of $R_{j}$ with the algebra of all $2 \times 2$ matrices that takes

$$
Q_{j} \rightarrow\left(\begin{array}{rr}
1 & 0 \\
0 & -1
\end{array}\right)
$$

and

$$
P_{j} \rightarrow\left(\begin{array}{rr}
0 & i \\
-i & 0
\end{array}\right)
$$

It is clear that $R_{j}$ is isomorphic to the Clifford algebra on a two-dimensional space and hence to a $2 \times 2$ matrix algebra (in a fashion preserving the adjoint as well as the usual algebraic operations). The unitary transformation $V^{(j)}$ therefore exists and is unique within multiplication by a scalar unitary matrix, which does not affect the factors in the infinite product.

To prove the corollary, note that the Clifford distribution on a Hilbert space may be obtained as the skew product of its restrictions to one-dimensional subspaces spanned by the respective $e_{j}$. Now the Clifford algebra in one dimension is spanned by elements $f_{0}$ and $f_{1}$ with $f_{0} f_{1}=f_{1} f_{0}=f_{1}$ and $f_{0}^{2}=f_{1}^{2}=f_{0}$ (taking the variance parameter of the distribution as unity, which is no essential loss of generality). It may be represented therefore as the direct sum of two copies of the complex number field, with $f_{0}=(1,1)$ and $f_{1}=(1,-1)$. In this representation the free-field $P$ and $Q$ defined above are readily computed to have the form: 


$$
P=2^{-(1 / 2)}\left(\begin{array}{rr}
0 & i \\
-i & 0
\end{array}\right)
$$

and

$$
Q=2^{-(1 / 2)}\left(\begin{array}{rr}
1 & 0 \\
0 & -1
\end{array}\right) \text {. }
$$

Now according to Theorem 5 the existence of a unitary operator $U$ with the stated property is equivalent to the convergence of $\prod_{j}\left|\left(V^{(j)} I, I\right)\right|^{2}$, where $I$ here denotes the identity element of the ring of the Clifford distribution (note that $\left.\left|\left(A_{\lambda}\left(P_{\lambda}\right) z_{\lambda}, z_{\lambda}\right)=\right|\left(U_{\lambda} z_{\lambda}, z_{\lambda}\right)\right|^{2}$ in case the automorphism $A_{\lambda}$ is induced by the unitary operator $\left.U_{\lambda}\right)$. From the rules $E\left(f_{0}^{2}\right)=E\left(f_{1}^{2}\right)=1$ while $E\left(f_{1}\right)=0$, it follows that the inner product on the Clifford algebra in the above representation has the form $\left(\left(\alpha_{1}, \alpha_{2}\right),\left(\gamma_{1}, \gamma_{2}\right)\right)=\left(\alpha_{1} \gamma_{1}+\alpha_{2} \gamma_{2}\right) / 2$. Now $\left(V^{(j)} I, I\right)=\left(W^{(j)} f_{0}, f_{0}\right)$, where $W^{(j)}$ is the matrix $\left(w_{r s}^{(j)}\right)$, and evaluating the indicated inner product it results that the $j$ th factor in the product given in Corollary 5.3 is precisely the square root of the $j$ th factor in the product given in Theorem 5.

EXAmPLE 3.1. Consider the transformation $P_{j} \rightarrow P_{j}^{\prime}=P_{j} \cos \theta_{j}-Q_{j} \sin \theta_{j}$, $Q_{j} \rightarrow Q_{j}^{\prime}=P_{j} \sin \theta_{j}+Q_{j} \cos \theta_{j}(j=1,2, \cdots)$. It is readily checked that the transformation is pseudo-canonical (i.e. the $P_{j}^{\prime}$ and $Q_{j}^{\prime}$ satisfy the FermiDirac anti-commutation relations). In fact one computes without difficulty that $W^{(j)}$ may be taken as

$$
\left(\begin{array}{cc}
\cos \left(\theta_{j} / 2\right) & i \sin \left(\theta_{j} / 2\right) \\
i \sin \left(\theta_{j} / 2\right) & \cos \left(\theta_{j} / 2\right)
\end{array}\right) .
$$

The transformation is therefore implementable by a unitary operator if and only if the product $\prod_{j}\left|\left(\cos \left(\theta_{j} / 2\right)+i \sin \left(\theta_{j} / 2\right)\right)\right|$ is convergent, which is always the case. It is easy to describe rather explicitly in this case the transforming unitary: it is the duality transform of the unitary transformation on the skewsymmetric tensors over the complex extension $\mathcal{F}^{c}$ of $\mathcal{H}$, that is induced by the unitary transformation on $H^{c}$ that takes $e_{j}$ into $\exp \left(i \theta_{j} / 2\right) e_{j}$ $(j=1,2, \cdots)$.

ExAmple 3.2. In the case of the transformation $P_{j} \rightarrow-P_{j}, Q_{j} \rightarrow Q_{j}, W^{(j)}$ may be taken as

$$
\left(\begin{array}{rr}
1 & 0 \\
0 & -1
\end{array}\right)
$$

and all the factors in the given product vanish, so that the transformation can not be effected by a unitary equivalence. Thus a second proof is obtained for the inequivalence of the free-field and anti-free-field systems described in connection with Theorem 7 . It also follows that the transformation $P_{j} \rightarrow Q_{j}$, 
$Q_{j} \rightarrow P_{j}$, is not canonical, in the strict sense.

EXAmple 3.3. In the case of the transformation $P_{j} \rightarrow i 2^{(1 / 2)} P_{j} Q_{j}, Q_{j} \rightarrow Q_{j}$, the transforming matrix $W^{(j)}$ may be taken as

$$
\left(\begin{array}{ll}
1 & 0 \\
0 & i
\end{array}\right)
$$

and it follows that the indicated transformation is not strictly canonical.

ExAmple 3.4. As in the Bose-Einstein case, the method involved in Corollary 5.3 applies whenever the distribution is represented as the skew product of distributions on finite-dimensional subspaces; it is not necessary that the subspaces be one-dimensional. On the other hand, it is not at all essential to make use of explicit isomorphisms between Clifford numbers and matrices. The following example illustrates both of these points. It is pertinent to the bilinear interaction between fermions, and indicates that as in the case of bosons, there exists no actual $S$-operator transforming the uncoupled into the coupled field operators.

Let $\mathfrak{H}^{C}=\mathfrak{F}^{(1)}+\mathcal{F}^{(-1)}$, where $\mathfrak{H}^{(1)}$ and $\mathfrak{F}^{(-1)}$ are isomorphic in a distinguished fashion, which takes the basis $\left\{e_{j}^{(1)}\right\}$ of $\mathfrak{F}^{(1)}$ into that $\left\{e_{j}^{(-1)}\right\}$ of $\mathfrak{F C}^{(-1)}$. Set $Q_{j}^{(k)}=Q\left(e_{j}^{(k)}\right)$ and $P_{j}^{(k)}=P\left(e_{j}^{(k)}\right)$, where $(P(\cdot), Q(\cdot))$ is the free-field system over FC. Now consider the following transformation: $P_{j}^{(k)} \rightarrow P_{j}^{(k) \prime}=P_{j}^{(k)}$, while

$$
Q_{j}^{(k)} \rightarrow Q_{j}^{(k) \prime}=Q_{j}^{(k)} \cos \theta+Q_{j}^{(-k)} \sin \theta .
$$

(In a formal manner, which is quite unrigorous, except in the relatively trivial case that $H$ is finite-dimensional, this transformation is obtained by transforming the free-field system by the formally unitary, but actually generally nonexistent, operator $S=\exp \left(\theta \sum_{j} Q_{j}^{(1)} Q_{j}^{(-1)}\right)$.)

We apply Theorem 5 to the decomposition of $\mathfrak{F}$ as the direct sum of the two-dimensional subspaces spanned by $e_{j}^{(1)}$ and $e_{j}^{(-1)}$. The Clifford algebra over such a two-dimensional subspace is generated by elements $f_{0}$, and $f_{ \pm 1}$, such that $f_{0} f_{ \pm 1}=f_{ \pm 1} f_{0}=f_{ \pm 1}, f_{ \pm 1}^{2}=f_{0}^{2}=f_{0}$, and $f_{1} f_{-1}=-f_{-1} f_{1}$. The corresponding $Q^{\prime}$ s are then $Q^{ \pm 1}: u \rightarrow 2^{-(1 / 2)} f_{ \pm 1} u$, where $u$ is arbitrary in the Clifford algebra. The indicated transformation $Q^{ \pm 1} \rightarrow Q^{ \pm 1^{\prime}}, P^{ \pm 1} \rightarrow P^{ \pm 1^{\prime}}\left(=P^{ \pm 1}\right)$ may be effected as follows: $T \rightarrow R^{*} T R$, where $R$ is the operation $u \rightarrow r \cdot u$, and $r=\cos (\theta / 2)$ $+f_{1} f_{-1} \sin (\theta / 2)$. Thus implementability of the given transformation by a unitary operator is equivalent to the convergence of $\prod_{j} \gamma_{j}$, where $\gamma_{j}$ $=\left|\left(R f_{0}, f_{0}\right)\right|$. Now $\left(R f_{0}, f_{0}\right)=E(R)$ (since $R$ is in the algebra generated by the $\left.Q^{ \pm 1}\right)=\operatorname{tr}(r)=\cos (\theta / 2)$. Thus it is only in the trivial case when $\theta$ is congruent to zero modulo $2 \pi$ that the interacting (scattered) field canonical system is unitarily equivalent to the free-field system.

\section{REFERENCES}

1. R. H. Cameron and W. T. Martin, Transformation of Wiener integrals under translations, Ann. of Math. vol. 45 (1944) pp. 386-396. 
2. - Transformation of Wiener integrals under a general class of linear transformations, Trans. Amer. Math. Soc. vol. 58 (1945) pp. 184-219.

3. P. A. M. Dirac, The principles of quantum mechanics, 3d ed., Oxford, 1947.

4. K. O. Friedrichs, Mathematical aspects of the quantum theory of fields, New York, 1953.

5. L. Gårding and A. S. Wightman, Representations of the anticommutation relations, Proc. Nat. Acad. Sci. U.S.A. vol. 40 (1954) pp. 617-621.

6. - Representations of the commutation relations, Proc. Nat. Acad. Sci. U.S.A. vol. 40 (1954) pp. 622-626.

7. S. Kakutani, On equivalence of infinite product measures, Ann. of Math. vol. 49 (1948) pp. 214-224.

8. F. J. Murray and J. von Neumann, On rings of operators IV, Ann. of Math. vol. 44 (1943) pp. 716-808.

9. I. E. Segal, Decompositions of operator algebras. II, Memoirs of the American Mathematical Society, no. 9, New York 1951.

10. - A non-commutative extension of abstract integration, Ann. of Math. vol. 57 (1953) pp. 401-457.

11. - Tensor algebras over Hilbert spaces. I, Trans. Amer. Math. Soc. vol. 81 (1956) pp. 106-134.

12. ——, Tensor algebras over Hilbert spaces. II, Ann. of Math. vol. 63 (1956) pp. 160175.

13. - Convergent approach to elementary particle interactions, Proc. Nat. Acad. Sci. U.S.A. vol. 42 (1956) pp. 670-676.

14. - Ergodic subgroups of the orthogonal group on a real Hilbert space, Ann. of Math. vol. 66 (1957) pp. 297-303.

15. J. von Neumann, Die eindeutigkeit der Schrödingerschen Operatoren, Math. Ann. vol. 104 (1931) pp. 570-578.

16. — On infinite direct products, Compositio Math. vol. 6 (1938) pp. 1-77.

University of ChicaGo,

Chicago, Ill.

InSTITUTE For Advanced StUdy,

Princeton, N. J. 